\title{
Cellular Physiology

\section{Gender-dependent Protein Expression in White Adipose Tissues of Lean and Obese Rats Fed a High Fat Diet}

\author{
Rajib Mukherjee, Jung-Won Choi, Duk Kwon Choi, Tae Seok Oh, \\ Hao Liu and Jong Won Yun
}

Department of Biotechnology, Daegu University, Kyungsan, Kyungbuk

\section{Key Words}

2-DE • Gender difference $\cdot$ High fat diet $•$ Proteome $•$ White adipose tissue

\begin{abstract}
Background: Proper understanding of molecular mechanisms underlying gender dimorphism in obesity for better nutritional recommendation is still in early stages. As white adipose tissues (WAT) is most important tissue in obesity metabolism, comparative proteomic analysis of all three WAT deposits at the same time to yield immensely important protein markers was the primary goal of this study. Methods: We performed differential expression analysis of protein profiles of three different WAT viz. subcutaneous, inguinal, and abdominal fat deposits of both genders in lean and obese rats fed a high fat diet (HFD) using a combination of 2-DE and MALDITOF-MS. Results: The proteomics analysis enabled us to detect 25, 29, and 46 proteins showing gender differences in three WAT deposits, respectively, to gain insight into cause of higher body weight gain in male in response to HFD. Conclusion: The gender dimorphism found in this proteomic study implies that female rats have a lower tendency to undergo
\end{abstract}

\section{KARGER}

Fax +41613061234

E-Mail karger@karger.ch

www.karger.com
(C) 2012 S. Karger AG, Basel

$1015-8987 / 12 / 0294-0617 \$ 38.00 / 0$

Accessible online at:

www.karger.com/cpb metabolic syndrome manifestation, which is associated with lower reliance on lipid as an energy fuel, lower lipogenesis, as well as increased mitochondrial oxidative capacity. In conclusion, most of the candidate proteins identified herein by differential proteomics were previously unrecognized in gender dimorphism of adipose tissue.

Copyright $\odot 2012$ S. Karger AG, Base

\section{Introduction}

Obesity has become a major health problem throughout developed as well as undeveloped countries, and leads primarily to other chronic metabolic diseases such as atherosclerosis and diabetes $[1,2]$. Currently, more than one billion adults are overweight, and at least 300 million are clinically obese (WHO, 2009). Obesity is commonly known as the storage of fat that is hazardous to one's health and obesity can result in hypertrophy as well as hyperplasia of adipocytes.

Adipose tissues are the most important tissue to the progression of obesity, and white adipose tissue (WAT)

Dr. Jong Won Yun

Department of Biotechnology, Daegu University, Kyungsan

Kyungbuk 712-714 (Republic of Korea)

Tel. +82-53-850-6556, Fax +82-53-850-6559

E-Mail jwyun@daegu.ac.kr 
has been shown to play a major role in the development of obesity. WAT acts as an active endocrine organ and secrets signaling molecules termed as adipokines that are involved in the regulation of food intake, energy homeostasis as well as inflammatory conditions associated with several metabolic complication $[3,4]$.

One common phenotype related to mammalian obesity is gender differences, in terms of the development of obesity as well as fat distribution in the body [5]. It is well recognized that gender differences in obesity originate mainly from metabolic and hormonal differences between the sexes [6]. The differential development of obesity in males and females result from the social and cultural factors, but the most profound cause is biological factors including differences in body fat distribution, energy balance, and hormonal effect [7-10]. However, gender differences in obesity and other metabolic disorders cannot be explained simply by the action and presence of sex hormones in a gender-different manner [11]. Although it has been hypothesized that sex is a strong influencing factor in health and illness, development of gender-specific medication is still in its early age [12]. Broader gendersensitive knowledge of metabolic diseases like obesity provide basic interventions and allow prevention as well as treatment by matching different needs of men and women such as the development of gender-specific medicine and various health policies [13].

In our previous studies, we investigated genderdependent protein expression in plasma [14], skeletal muscle [15], brown adipose tissue [16], and liver [17] of rats in response to HFD treatment and found existence in clear gender dimorphism. To date, proteome profiling of adipose tissues has been established by many investigators, focusing mostly on a specific fat deposit [18-20]. However, due to their diverse physiological roles in metabolic syndromes, a separate proteome profiling against different fat deposits is needed. We therefore analyzed the proteomes of three different types of WAT viz. subcutaneous, inguinal, and abdominal WAT, and attempted to identify marker proteins in relation to obesity with special attention to gender dimorphism from each WAT.

\section{Materials and Methods}

\section{Animals and breeding conditions}

Male and female SLC Sprague-Dawley (SD) rats were purchased from Daehan Experimental Animals (Seoul, Korea) at 5 weeks of age, and were housed one per cage in a temperature $\left(23 \pm 2^{\circ} \mathrm{C}\right)$ and humidity $(55 \%)$-controlled room with a $12 \mathrm{~h}$ light/

\begin{tabular}{lcc}
\hline Ingredient & ND & $\begin{array}{c}\text { HFD } \\
\text { Composition by weight, g/kg }\end{array}$ \\
\hline Casein & 200 & 200 \\
Cornstarch & 150 & 155 \\
Sucrose & 500 & 50 \\
Dextrose & 0 & 132 \\
Cellulose & 50 & 50 \\
Soybean oil & 0 & 25 \\
Corn oil & 50 & 0 \\
Lard & 0 & 175 \\
Mineral mix & 35 & 35 \\
Vitamin mix ${ }^{\text {b) }}$ & 10 & 10 \\
TBHQ & 0 & 0.014 \\
DL-Methionin & 3 & 0 \\
L-cystine & 0 & 3 \\
Choline bitartrate & 2 & 2.5 \\
& & Composition by calories, $\%$ \\
Protein & 21 & 20 \\
Carbohydrate & 68 & 35 \\
Fat & 12 & 45 \\
Total & $3902 \mathrm{kcal} / \mathrm{kg}$ & $4776 \mathrm{kcal} / \mathrm{kg}$ \\
\hline
\end{tabular}

Table 1. Composition of diets used in this study. a)Mineral mix; sucrose $(118.03 \mathrm{~g} / \mathrm{kg})$, calcium phosphate dibasic $(500 \mathrm{~g} /$ $\mathrm{kg}$ ), sodium chloride $(74 \mathrm{~g} / \mathrm{kg})$, potassium citrate $(220 \mathrm{~g} / \mathrm{kg})$, potassium sulfate $(52 \mathrm{~g} / \mathrm{kg})$, magnesium oxide $(24 \mathrm{~g} / \mathrm{kg})$, magnesium oxide $(24 \mathrm{~g} / \mathrm{kg})$, manganous carbonate $(3.5 \mathrm{~g} / \mathrm{kg})$, ferric citrate $(6 \mathrm{~g} / \mathrm{kg})$, zinc carbonate $(1.6 \mathrm{~g} / \mathrm{kg})$, cupric carbonate $(0.3 \mathrm{~g} / \mathrm{kg})$, potassium iodate $(0.01 \mathrm{~g} / \mathrm{kg})$, sodium selenite $(0.01$ $\mathrm{g} / \mathrm{kg})$, chromium potassium sulfate $(0.55 \mathrm{~g} / \mathrm{kg})$. b) Vitamine mix, sucrose $(981.15 \mathrm{~g} / \mathrm{kg})$, thiamine $(0.6 \mathrm{~g} / \mathrm{kg})$, vitamin $\mathrm{E}$ acetate $(500 \mathrm{IU} / \mathrm{g} ; 10 \mathrm{~g} / \mathrm{kg})$, niacin $(3 \mathrm{~g} / \mathrm{kg})$, calcium pantothenate $(1.6$ $\mathrm{g} / \mathrm{kg})$, vitamin B12 $(0.1 \% ; 1 \mathrm{~g} / \mathrm{kg})$, vitamin A palmitate $(500,000$ $\mathrm{IU} / \mathrm{g} ; 0.8 \mathrm{~g} / \mathrm{kg})$, pyridoxine $(0.7 \mathrm{~g} / \mathrm{kg})$, riboflavin $(0.6 \mathrm{~g} / \mathrm{kg})$, vitamin D3 $(400,000 \mathrm{IU} / \mathrm{g} ; 0.25 \mathrm{~g} / \mathrm{kg})$, folic acid $(0.2 \mathrm{~g} / \mathrm{kg})$, menadione sodium bisulfate $(0.08 \mathrm{~g} / \mathrm{kg})$, biotin $(0.02 \mathrm{~g} / \mathrm{kg})$. c)TBHQ, tert-butylhydroquinone.

dark cycle. Rats were provided free access to standard chow and tap water for an adaptation period of 1 week. Male and female rats were randomly divided into two groups, where 20 rats were fed a normal diet (ND, 12\% calories from fat; control group) and 20 rats were fed a high fat diet (HFD, 45\% calories from fat). Rat foods were purchased from Feed Korea Lab (Hanam, Korea) and the dietary composition of these feeds is shown in Table 1. All rats and foods were weighed every week for 8 weeks. Rats were food deprived for at least $12 \mathrm{~h}$ before

Mukherjee/Choi/Choi/Oh/Liu/Yun 
being sacrificed and were anesthetized with 3\% diethyl ether. These experiments were approved by the Committee for Laboratory Animal Care and Use of Daegu University. All procedures were conducted in accordance with the Guide for the Care and Use of Laboratory Animals published by the National Institutes of Health.

Preparation of WAT sample for two-dimensional electrophoresis (2-DE)

The rats were fasted overnight and anesthetized using diethyl ether prior to sacrifice. After sacrifice, inguinal, abdominal, and subcutaneous WAT were immediately collected and washed in saline water, pulverized in liquid nitrogen and finally stored in $-80^{\circ} \mathrm{C}$. For the 2-DE experiments the tissues were homogenized in $300 \mu 1$ rehydration buffer containing $7 \mathrm{M}$ urea, $2 \mathrm{M}$ thiourea, 4\% CHAPS, $20 \mathrm{mM}$ DTT, $1 \mathrm{mM} \mathrm{PMSF,} 2 \%$ IPG buffer (ampholyte 3/10, Bio-Rad) and a trace of bromophenol blue with homogenizer (PT 1200E, Kinematica Ltd., Luzern, Switzerland) on ice. The homogenate was then centrifuged for $15 \mathrm{~min}$ at $14000 \mathrm{xg}$ followed by TCA precipitation method. After the protein pellet was dried, it was resuspended in $200 \mu \mathrm{l}$ rehydration buffer. The protein content was calculated quantitatively by the Bradford method [21]. After quantitative protein measurement, the proteins samples were pooled by mixing $150 \mu \mathrm{g}$ from each of the 7 protein sample. This mixture was then used as protein sample for further 2-DE experiments.

\section{$2-D E$}

2-DE was performed three times using each pooled WAT protein samples from seven rats per group, which consisted of the control group, who were fed an ND, and the obese group, who were fed an HFD. The same diet was provided to both male and female rats. 2-DE experiments were conducted using previously described methods [22]. Briefly, immobilized $\mathrm{pH}$ gradient (IPG)-isoelectric focusing (IEF) of WAT samples was performed on $\mathrm{pH}$ 3-10 and $18 \mathrm{~cm}$ IPG DryStrips (GE Healthcare, Buckinghamshire, UK) in a PROTEAN IEF cell (Bio-Rad) using the protocol recommended by the manufacturer. IPG strips were rehydrated passively for $24 \mathrm{~h}$ in strip holders with $350 \mu \mathrm{L}$ of rehydration solution, which included $7 \mathrm{M}$ urea (Bio Basic, Ontario, Canada), $2 \mathrm{M}$ thiourea (Sigma-Aldrich, St. Louis, MO, USA), 4\% CHAPS (Bio Basic), 1 mM PMSF (Sigma-Aldrich), 20 mM DTT (GE Healthcare), and 2\% IPG buffer (Bio-Rad), and WAT protein of $150 \mu \mathrm{g}$. IEF was conducted as follows: $15 \mathrm{~min}$ at $250 \mathrm{~V}, 3 \mathrm{hr}$ at $250-10,000 \mathrm{~V}, 6 \mathrm{hr}$ at $10,000 \mathrm{~V}$, and then held at $500 \mathrm{~V}$ until ready to run the second dimension. After focusing, gel strips were equilibrated in a solution containing $6 \mathrm{M}$ urea, 2\% SDS (Generay Biotech, Shanghai, China), 1\% DTT, 30\% glycerol (Bio Basic), and $50 \mathrm{mM}$ Tris- $\mathrm{HCl}$ (pH 6.8) for $20 \mathrm{~min}$, followed by further incubation in the same solution, except DTT was replaced with the $2.5 \%$ iodoacetamide (Bio-Rad) for an additional $20 \mathrm{~min}$. Gel strips were then placed onto a 20x20 $\mathrm{cm} 12 \%$ polyacrylamide gel for resolution in the second dimension. Fractionation was performed using the Laemmli SDSdiscontinuous system at a constant voltage of $15 \mathrm{~mA}$ per gel for $14 \mathrm{~h}$. For image analysis and peptide mass fingerprinting (PMF), a total of 36 gels, including 3 gels per group with separated proteins were visualized by silver staining. Silver

Gender-dependent Protein Expression in White Adipose Tissues staining was performed as follows. Gels were fixed over a period of $30 \mathrm{~min}$ in $50 \%$ ethanol (Duksan Pure Chemicals, Ansan, Korea) and 5\% acetic acid (Duksan Pure Chemicals), followed by $10 \mathrm{~min}$ in $30 \%$ ethanol. The gels were then washed with water for $5 \mathrm{~min}$ three times. Gels were sensitized for $10 \mathrm{~min}$ in $0.02 \%$ sodium thiosulfate (Sigma-Aldrich), followed by $0.5 \mathrm{~min}$ washes in water three times, and incubated for $25 \mathrm{~min}$ in $0.3 \%$ silver nitrate (Kojima Chemicals, Sayama, Japan). After two 0.5 min water washes, proteins were visualized using the developing solution (3\% sodium carbonate; Duksan Pure Chemicals), $0.02 \%$ sodium thiosulfate, $0.05 \%$ formalin (DC Chemicals, Incheon, Korea), and stopped with $6 \%$ acetic acid.

\section{Image acquisition and data analysis}

Gels were imaged on a UMAX PowerLook 1120 (Maxium Technologies, Akron, OH, USA) and the images were compared using modified ImageMaster 2-D software V4.95 (GE Healthcare). Protein spots were automatically detected using the software and the spots were edited manually if necessary. For each experiment, a reference gel was selected from gels of the normal group and spots detected from the other gels were matched to those in the reference gel. Relative optical density and relative volume were also calculated to correct for differences in gel staining. Each spot intensity volume was processed by background subtraction and total spot volume normalization. The resulting spot volume percentage (\%Volume) was used for comparison as described previously [23, 24].

\section{Protein identification}

For protein identification, protein spots were excised, digested with trypsin (Promega, Madison, WI), mixed with $\alpha$ cyano-4-hydroxycinnamic acid in 50\% acetonitrile/ $0.1 \%$ trifluoroacetic acid, and subjected to matrix-assisted laser desorption/ionization-time-of-flight (MALDI-TOF) analysis (Ettan MALDI-TOF Pro, GE Healthcare). Spectra were collected from 350 shots per spectrum over a $\mathrm{m} / \mathrm{z}$ range of 600-3000 and calibrated by two point internal calibration using trypsin autodigestion peaks $(\mathrm{m} / \mathrm{z} 842.5099,2211.1046)$. Peak lists were generated using the Ettan MALDI-TOF Pro Evaluation Module (ver. 2.0.16). The threshold used for peak-picking was as follows: 5,000 for minimum resolution of monoisotopic mass and 2.5 for $\mathrm{S} / \mathrm{N}$. The search program MASCOT (Mascot Sever 2.3), which was developed by the Matrixscience (http:// www.matrixscience.com), was used for protein identification by PMF. The following parameters were used for the database search: trypsin as the cleaving enzyme, a maximum of one missed cleavage, iodoacetamide (Cys) as a fixed modification, oxidation (Met) as a variable modification, monoisotopic masses, and a mass tolerance of $\pm 0.1 \mathrm{Da}$. The MASCOT probability-based MOWSE (molecular weight search) score was calculated for PMF. The protein score was $-10 * \log (\mathrm{P})$, where $\mathrm{P}$ was the probability that the observed match was a random event, and greater than 61 was considered significant $(p<0.05)$.

\section{Immunoblot analysis}

The levels of five proteins identified in the 2-DE protein map of three WAT types were confirmed by immunoblot 


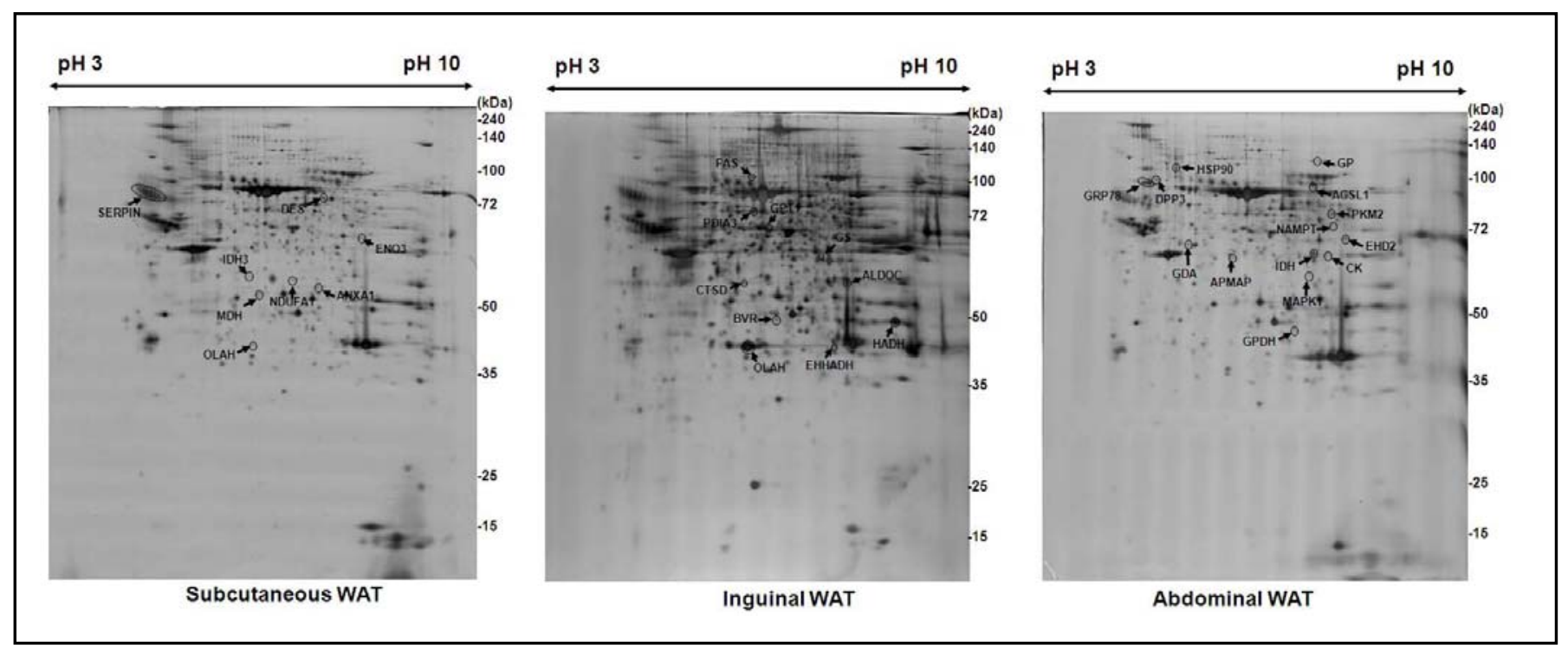

Fig. 1. Representative silver-stained 2-DE gel images of rat subcutaneous, inguinal, and abdominal WAT proteome in HFD rats. Proteins showing gender differences are marked with arrows and their abbreviated names. For the full name of each protein, see Table 2.

analysis. Tissue lysates were prepared with RIPA buffer (SigmaAldrich), homogenized, and centrifuged at $12,000 \mathrm{xg}$ for $20 \mathrm{~min}$. The extract was diluted in 5X sample buffer $(50 \mathrm{mM}$ Tris of $\mathrm{pH}$ $6.8,2 \%$ SDS, $10 \%$ glycerol, $5 \% \beta$-mercaptoethanol, and $0.1 \%$ bromophenol blue) and heated for $5 \mathrm{~min}$ at $95^{\circ} \mathrm{C}$ before SDSpolyacrylamide gel electrophoresis (PAGE) using $6,8,10$, or $12 \%$ acrylamide gels. After electrophoresis, samples were transferred to a polyvinylidene difluoride (PVDF, Santa Cruz Biotechnology, Santa Cruz, CA, USA) membrane and blocked for $1 \mathrm{~h}$ with TBS (Tris-buffered saline)-T buffer (10 mM Tris$\mathrm{HCl}, 150 \mathrm{mM} \mathrm{NaCl}, 0.1 \%$ Tween 20 containing 5\% skim milk). The membrane was rinsed with 3 changes of TBS-T buffer, followed by incubation for $1 \mathrm{~h}$ with a 1:1000 dilution of primary monoclonal antibody anti- $\beta$-actin, anti-MDH, anti-EHD2, antiCK, anti-GP, (Santa Cruz Biotechnology), and anti-FAS (Cell Signaling Technology, Beverly, MA, USA) in TBS-T buffer containing $1 \%$ skim milk. After 3 washes, the membrane was incubated for $1 \mathrm{~h}$ with horseradish peroxidase-conjugated antigoat IgG, anti-mouse IgG, or anti-rabbit IgG secondary antibody (1:1000, AB Frontier, Seoul, Korea) in TBS-T buffer containing $1 \%$ skim milk and developed using enhanced chemiluminescence (ECL; GE Healthcare). Immunoblot analysis was performed by scanning with a UMAX PowerLook 1120 and digitalized using image analysis software (KODAK 1D, Eastman Kodak, Rochester, NY, USA).

\section{Statistical analysis}

All experimental results were compared by one-way analysis of variance (ANOVA) using the Statistical Package for Social Science (SPSS, version 14.0K) program and data were expressed as the mean $\pm \mathrm{SD}$. Group means were considered significantly different at $p<0.05$, as determined by the protected least-significant difference (LSD) when ANOVA indicated an overall significant treatment effect $(p<0.05)$.

\section{Results}

HFD-induced phenotypes in male and female rats

At the beginning, body weight and food intake of ND and HFD rats were nearly identical for both male and female rats (data not shown, see ref. [14]). However, after 2 weeks, body weight and food intake of the HFD rats were higher $(p<0.05)$ than those of ND rats at all subsequent time points in both genders [14]. Total body gain per food intake of HFD fed male rats was higher in female by an average of approximately $30 \%(p=0.001)$. In addition, body weight and food intake were found to be significantly higher in male rats than in female rats in both groups [14]. Differences in food efficiency of the rats were also observed between genders [14]. These collective results from animal experiments prompted us to perform further proteomic studies.

\section{Proteomic analysis of WAT proteins}

High throughput 2-DE-based proteomic approaches were conducted using pooled protein samples from seven rats for subcutaneous, inguinal and abdominal WAT type to obtain three gel replicas of each group. Each IEF $(\mathrm{pH}$ 3-10) strip was hydrated with $150 \mu \mathrm{g}$ protein and separated for the first dimension followed by $12 \%(\mathrm{w} / \mathrm{v})$ SDS-PAGE for the second dimension. Based on high scores $(p<0.05)$ and sequence coverage, a total of 25,29 , and 46 proteins showing differential expression between the genders and/ 


\begin{tabular}{|c|c|c|c|c|c|c|c|c|}
\hline \multirow[b]{2}{*}{ Protein } & \multirow[b]{2}{*}{ Acc. No. ${ }^{1}$} & \multirow[b]{2}{*}{$\begin{array}{l}\text { Nominal mass } \\
(\mathrm{Mr})^{2)}\end{array}$} & \multirow[b]{2}{*}{$\begin{array}{l}\text { Calculated } \\
\text { pI }\end{array}$} & \multicolumn{2}{|c|}{$\mathrm{ND}^{3)}$} & \multicolumn{2}{|c|}{$\mathrm{HFD}^{31}$} & \multirow[b]{2}{*}{ Score $^{51}$} \\
\hline & & & & $\begin{array}{l}\text { Males }{ }^{4)} \\
(\% \text { vol })\end{array}$ & $\begin{array}{l}\left.\text { Females }{ }^{4}\right) \\
(\% \text { vol })\end{array}$ & $\begin{array}{l}\left.\text { Males }{ }^{4}\right) \\
(\% \text { vol })\end{array}$ & $\begin{array}{l}\text { Females }{ }^{4)} \\
(\% \text { vol })\end{array}$ & \\
\hline \multicolumn{9}{|c|}{ Group I: Gender different in both ND and HFD rats with higher protein abundances in males } \\
\hline \multicolumn{9}{|l|}{ Subcutaneous WAT } \\
\hline Zero beta globulin & gi 802111 & 15953 & 6.81 & $0.73 \pm 0.13$ & $0.32 \pm 0.08 *$ & $0.44 \pm 0.08$ & $0.26 \pm 0.05^{*}$ & 68 \\
\hline Desmin (DES) & gi38197676 & 53447 & 5.21 & $0.15 \pm 0.01$ & $0.06 \pm 0.01 * *$ & $0.22 \pm 0.01$ & $0.13 \pm 0.02 * *$ & 226 \\
\hline Serine/cysteine peptidase inhibitor (SERPIN) & $\operatorname{gi} 133777069$ & 45719 & 5.24 & $5.94 \pm 1.07$ & $2.08 \pm 0.38^{*}$ & $3.40 \pm 0.19$ & $1.87 \pm 0.23 * *$ & 123 \\
\hline \multicolumn{9}{|l|}{ lnguinal WAT } \\
\hline Hydroxyacyl CoA dehydrogenase (HADH) & gil7105336 & 34540 & 8.83 & $1.35 \pm 0.24$ & $0.64 \pm 0.11^{*}$ & $1.52 \pm 0.17$ & $0.70 \pm 0.10 * *$ & 137 \\
\hline Fructose bisphosphate aldolase C (ALDOC) & gil 334163 & 39658 & 6.78 & $0.40 \pm 0.10$ & $0.16 \pm 0.03^{*}$ & $0.25 \pm 0.03$ & $0.13 \pm 0.02 *$ & 115 \\
\hline Fatty acid synthase (FAS) & gi2506136 & 275101 & 5.96 & $0.13 \pm 0.01$ & $0.08 \pm 0.01^{*}$ & $0.18 \pm 0.04$ & $0.07 \pm 0.01^{*}$ & 73 \\
\hline Rat mitochondrial 3,2 enoyl- CoA & gi56967286 & 29418 & 8.91 & $0.55 \pm 0.08$ & $0.30 \pm 0.05^{*}$ & $0.91 \pm 0.21$ & $0.37 \pm 0.07^{*}$ & 62 \\
\hline $\begin{array}{l}\text { S acyl fatty acid synthase thioesterase } \\
\text { (OLAH) }\end{array}$ & gi 12083671 & 29623 & 5.80 & $1.97 \pm 0.17$ & $0.70 \pm 0.08 * *$ & $3.11 \pm 0.53$ & $1.02 \pm 0.46 * *$ & 109 \\
\hline NADH dehydrogenase (ubiquinone) & gi 157817227 & 30379 & 7.07 & $0.76 \pm 0.21$ & $0.31 \pm 0.14^{*}$ & $0.96 \pm 0.06$ & $0.45 \pm 0.21^{*}$ & 115 \\
\hline Ester hydrolase CII or 154 hom olog & gi62079139 & 35427 & 6.16 & $0.44 \pm 0.11$ & $0.20 \pm 0.07^{*}$ & $0.55 \pm 0.12$ & $0.11 \pm 0.03^{*}$ & 181 \\
\hline Carbonic anhydrase 2 & gi9506445 & 29267 & 6.89 & $0.49 \pm 0.03$ & $0.15 \pm 0.04 * *$ & $0.62 \pm 0.03$ & $0.13 \pm 0.04 * *$ & 81 \\
\hline \multicolumn{9}{|l|}{ Abdominal WAT } \\
\hline Tpil protein & gi38512111 & 27214 & 7.07 & $0.46 \pm 0.07$ & $0.22 \pm 0.10^{*}$ & $0.36 \pm 0.04$ & $0.25 \pm 0.02 *$ & 122 \\
\hline $\begin{array}{l}\text { Glycerol } 3 \text { phosphate dehydrogenase } \\
\text { (GPDH) }\end{array}$ & gi57527919 & 38112 & 6.16 & $0.17 \pm 0.01$ & $0.04 \pm 0.02 *$ & $0.37 \pm 0.07$ & $0.18 \pm 0.01 *$ & 88 \\
\hline EH domain containing protein 2 (EHD2) & gi 67846074 & 61370 & 6.12 & $0.13 \pm 0.03$ & $0.06 \pm 0.01^{*}$ & $0.22 \pm 0.00$ & $0.09 \pm 0.04 *$ & 112 \\
\hline Aryl sulfotransferase & gi55765 & 33422 & 6.41 & $0.14 \pm 0.02$ & $0.08 \pm 0.00^{*}$ & $0.11 \pm 0.00$ & $0.08 \pm 0.01 *$ & 90 \\
\hline NADH dehydrogenase & gi38304046 & 31852 & 5.44 & $0.12 \pm 0.03$ & $0.04 \pm 0.03 *$ & $0.18 \pm 0.02$ & $0.08 \pm 0.01 * *$ & 122 \\
\hline Dipeptidyl peptidase 3 (DPP3) & $\operatorname{gi} 16758578$ & 83386 & 5.12 & $0.10 \pm 0.02$ & $0.05 \pm 0.02^{*}$ & $0.15 \pm 0.03$ & $0.06 \pm 0.02^{*}$ & 80 \\
\hline Cysteine sulfinate decarboxylase & gil 193072901 & 55807 & 6.84 & $0.15 \pm 0.02$ & $0.05 \pm 0.01 * *$ & $0.18 \pm 0.04$ & $0.07 \pm 0.04 *$ & 143 \\
\hline Glucose regulated protein 78 (GRP78) & gi 25742763 & 72473 & 5.07 & $0.40 \pm 0.01$ & $0.19 \pm 0.03 * *$ & $0.34 \pm 0.03$ & $0.25 \pm 0.02 *$ & 155 \\
\hline L lactate dehydrogenase & gi 8393706 & 36712 & 8.45 & $0.66 \pm 0.07$ & $0.28 \pm 0.03 * *$ & $0.45 \pm 0.11$ & $0.15 \pm 0.02 *$ & 106 \\
\hline \multicolumn{9}{|c|}{ Group II: Gender different in both ND and HFD rats with higher protein abundances in females } \\
\hline \multicolumn{9}{|l|}{ Subcutaneous WAT } \\
\hline Ezrin & gill 7902245 & 54254 & 6.16 & $0.03 \pm 0.01$ & $0.15 \pm 0.04 *$ & $0.01 \pm 0.01$ & $0.06 \pm 0.01 *$ & 120 \\
\hline NADH dehydrogenase (NDUFA1) & gi 170295834 & 40753 & 7.64 & $0.03 \pm 0.01$ & $0.08 \pm 0.01 *$ & $0.04 \pm 0.02$ & $0.11 \pm 0.01 *$ & 193 \\
\hline Aflatoxin $\mathrm{B} 1$ aldehyde reductase & gi2 7527649 & 37970 & 6.10 & $0.02 \pm 0.00$ & $0.09 \pm 0.02 *$ & $0.02 \pm 0.02$ & $0.08 \pm 0.01^{*}$ & 64 \\
\hline Annexin $\mathrm{Al}$ ( ANXA1) & gi6978501 & 39147 & 6.97 & $0.04 \pm 0.00$ & $0.26 \pm 0.04 *$ & $0.08 \pm 0.03$ & $0.16 \pm 0.02 *$ & 154 \\
\hline Beta enolase (ENO3) & gi 126723393 & 47326 & 7.08 & $0.07 \pm 0.00$ & $0.32 \pm 0.06^{*}$ & $0.10 \pm 0.02$ & $0.29 \pm 0.05 *$ & 90 \\
\hline Malate dehydrogenase (MDH) & gi15100179 & 36631 & 6.16 & $0.23 \pm 0.08$ & $0.42 \pm 0.06^{*}$ & $0.13 \pm 0.04$ & $0.25 \pm 0.00^{*}$ & 80 \\
\hline Creatine kinase $M$ type & gi6671762 & 43246 & 6.58 & $0.08 \pm 0.02$ & $0.43 \pm 0.13^{*}$ & $0.21 \pm 0.02$ & $0.48 \pm 0.11^{*}$ & 147 \\
\hline \multicolumn{9}{|l|}{ Inguinal WAT } \\
\hline Albumin & gil 49033753 & 53060 & 6.72 & $0.05 \pm 0.01$ & $0.26 \pm 0.03 * *$ & $0.14 \pm 0.02$ & $0.25 \pm 0.05^{*}$ & 182 \\
\hline Vinculin & gi 157822133 & 117112 & 5.83 & $0.06 \pm 0.01$ & $0.25 \pm 0.07^{*}$ & $0.07 \pm 0.03$ & $0.23 \pm 0.03 * *$ & 175 \\
\hline $\begin{array}{l}\text { Protein disulfide isomerase associated } 3 \\
\text { (PDIA3) }\end{array}$ & gil 49023097 & 54123 & 7.10 & $0.28 \pm 0.00$ & $0.59 \pm 0.07 *$ & $0.32 \pm 0.02$ & $0.51 \pm 0.06 *$ & 100 \\
\hline Osteoglycin/Mimecan & gil 157824206 & 34390 & 5.85 & $0.10 \pm 0.03$ & $0.22 \pm 0.03 *$ & $0.08 \pm 0.01$ & $0.26 \pm 0.06^{*}$ & 93 \\
\hline Biliverdin reductase $A$ (BVR) & gi50925455 & 33658 & 5.97 & $0.09 \pm 0.04$ & $0.25 \pm 0.01 *$ & $0.11 \pm 0.01$ & $0.19 \pm 0.02 * *$ & 153 \\
\hline \multicolumn{9}{|l|}{ Abdominal WAT } \\
\hline Guanine deaminase (GDA) & $\operatorname{gil} 148747414$ & 51439 & 5.48 & $1.03 \pm 0.00$ & $0.14 \pm 0.01 *$ & $0.06 \pm 0.01$ & $0.19 \pm 0.01 * *$ & 232 \\
\hline Seryl am inoacyl tRNA synthase & gi 149025695 & 61838 & 6.43 & $0.03 \pm 0.01$ & $0.08 \pm 0.02 *$ & $0.04 \pm 0.00$ & $0.09 \pm 0.01^{*}$ & 86 \\
\hline Macrophage capping protein & gi61556900 & 39060 & 6.11 & $0.06 \pm 0.01$ & $0.14 \pm 0.03 *$ & $0.05 \pm 0.01$ & $0.11 \pm 0.02 *$ & 144 \\
\hline $\begin{array}{l}\text { Adipocyte plasma membrane associated } \\
\text { protein (APMAP) }\end{array}$ & gi777735352 & 42207 & 5.64 & $0.12 \pm 0.08$ & $0.42 \pm 0.13 *$ & $0.07 \pm 0.03$ & $0.30 \pm 0.08 *$ & 180 \\
\hline Serpin B6 & gi 40018548 & 43276 & 5.79 & $0.04 \pm 0.03$ & $0.19 \pm 0.06^{*}$ & $0.05 \pm 0.00$ & $0.13 \pm 0.02 *$ & 71 \\
\hline Isocitrate dehydrogenase ([DH) & gil 3928690 & 47047 & 6.53 & $0.24 \pm 0.04$ & $0.48 \pm 0.04 * *$ & $0.25 \pm 0.04$ & $0.47 \pm 0.02 * *$ & 160 \\
\hline Mitogen activated protein kinase 1 (MAPK 1 ) & gi6 6754632 & 41648 & 6.50 & $0.09 \pm 0.01$ & $0.13 \pm 0.00^{*}$ & $0.08 \pm 0.02$ & $0.14 \pm 0.03 *$ & 81 \\
\hline Ca binding protein & gi488838 & 47590 & 4.95 & $0.37 \pm 0.13$ & $0.76 \pm 0.18^{*}$ & $0.38 \pm 0.07$ & $0.70 \pm 0.02^{*}$ & 87 \\
\hline
\end{tabular}

continued

or diets among the 354,324 , and 454 spots were identified in subcutaneous, inguinal, and abdominal WAT, respectively (Fig. 1), with high confidence based on MALDI-TOF/MS and database searches (Table 2).

Gender-dependent Protein Expression in White Adipose Tissues

\section{Gender-dependent differential expressions of WAT proteins}

In each WAT type, the total spots were divided into five groups depending on gender specific expression

Cell Physiol Biochem 2012;29:617-634 


\begin{tabular}{|c|c|c|c|c|c|c|c|c|}
\hline \multirow[b]{2}{*}{ Protein } & \multirow[b]{2}{*}{ Acc. No. ${ }^{1)}$} & \multirow[b]{2}{*}{$\begin{array}{l}\text { Nominal mass } \\
(\mathrm{Mr})^{2)}\end{array}$} & \multirow[b]{2}{*}{$\begin{array}{l}\text { Calculated } \\
\text { pI }\end{array}$} & \multicolumn{2}{|c|}{$N D^{3)}$} & \multicolumn{2}{|c|}{$\mathrm{HFD}^{3)}$} & \multirow[b]{2}{*}{ Score ${ }^{51}$} \\
\hline & & & & $\begin{array}{l}\left.\text { Males }^{4}\right) \\
\text { (\% vol) }\end{array}$ & $\begin{array}{l}\text { Females }{ }^{4)} \\
(\% \text { vol })\end{array}$ & $\begin{array}{l}\text { Males }{ }^{4)} \\
\text { (\% vol) }\end{array}$ & $\begin{array}{l}\left.\text { Females }{ }^{4}\right) \\
(\% \text { vol })\end{array}$ & \\
\hline \multicolumn{9}{|c|}{ Group III: Gender different in both ND and HFD rats with opposite protein abundances between the genders } \\
\hline \multicolumn{9}{|l|}{ Subcutaneous WAT } \\
\hline Actin related protein 3 homolog & gil61728791 & 47652 & 5.61 & $0.19 \pm 0.03$ & $0.07 \pm 0.04^{*}$ & $0.05 \pm 0.03$ & $0.17 \pm 0.01^{*}$ & 69 \\
\hline \multicolumn{9}{|l|}{ Inguinal WAT } \\
\hline Cathepsin D (CTSD) & gi1 15720 & 45165 & 6.66 & $0.27 \pm 0.08$ & $0.09 \pm 0.03 *$ & $0.06 \pm 0.02$ & $0.15 \pm 0.01^{* *}$ & 75 \\
\hline Rat enoyl CoA hydratase (EHHADH) & gi24159081 & 28498 & 6.41 & $0.45 \pm 0.06$ & $0.15 \pm 0.05^{* *}$ & $0.13 \pm 0.01$ & $0.19 \pm 0.02 *$ & 110 \\
\hline \multicolumn{9}{|l|}{ Abdominal WAT } \\
\hline Long chain fatty acid CoA ligase 1 (ACSL1) & gi 25742739 & 79155 & 6.60 & $0.19 \pm 0.02$ & $0.07 \pm 0.01 * *$ & $0.09 \pm 0.02$ & $0.17 \pm 0.00^{*}$ & 231 \\
\hline Glycogen phosphorylase (GP) & gil 158138498 & 97740 & 6.65 & $0.05 \pm 0.00$ & $0.01 \pm 0.00 * *$ & $0.01 \pm 0.00$ & $0.02 \pm 0.00 * *$ & 69 \\
\hline Creatine kinase $\mathrm{M}$ type $(\mathrm{CK})$ & gi6671762 & 43246 & 6.58 & $0.53 \pm 0.10$ & $0.22 \pm 0.07 *$ & $0.08 \pm 0.03$ & $0.67 \pm 0.14^{*}$ & 202 \\
\hline Beta enolase & gi126723393 & 47326 & 7.08 & $0.42 \pm 0.11$ & $0.15 \pm 0.05 *$ & $0.05 \pm 0.01$ & $0.33 \pm 0.02 * *$ & 101 \\
\hline $\begin{array}{l}\text { Pyruvate kinase isozyme M1/M2 } \\
\text { (PKM1/M2) }\end{array}$ & gil 6757994 & 58294 & 6.63 & $0.19 \pm 0.02$ & $0.10 \pm 0.04^{*}$ & $0.02 \pm 0.00$ & $0.18 \pm 0.04^{*}$ & 132 \\
\hline Moesin & gi149042266 & 67899 & 6.12 & $0.14 \pm 0.04$ & $0.05 \pm 0.01^{*}$ & $0.04 \pm 0.00$ & $0.05 \pm 0.00^{*}$ & 247 \\
\hline \multicolumn{9}{|l|}{ Group IV: Gender-different only in HFD rats } \\
\hline \multicolumn{9}{|l|}{ Subcutaneous WAT } \\
\hline Hemopexin & gi122065203 & 52060 & 7.58 & $0.58 \pm 0.17$ & $0.05 \pm 0.04$ & $0.76 \pm 0.13$ & $0.38 \pm 0.12 *$ & 169 \\
\hline Glutamine synthetase (GS) & gil 42349612 & 42982 & 6.64 & $0.29 \pm 0.13$ & $0.24 \pm 0.02$ & $0.35 \pm 0.04$ & $0.12 \pm 0.01^{* *}$ & 172 \\
\hline Isocitrate dehydrogenase $3\left(\mathrm{NAD}^{+}\right)(\mathrm{IDH} 3)$ & gil 149041704 & 39409 & 5.87 & $0.07 \pm 0.02$ & $0.09 \pm 0.02$ & $0.03 \pm 0.02$ & $0.18 \pm 0.06^{*}$ & 87 \\
\hline $\begin{array}{l}\text { S acyl fatty acid synthase thioesterase } \\
(\mathrm{OLAH})\end{array}$ & gi12883671 & 29623 & 5.80 & $0.28 \pm 0.06$ & $0.26 \pm 0.04$ & $0.06 \pm 0.01$ & $0.53 \pm 0.13^{*}$ & 83 \\
\hline Rat annexin $\mathrm{V}$, chain $\mathrm{A}$ & gi1 157836327 & 35458 & 4.97 & $0.06 \pm 0.02$ & $0.06 \pm 0.06$ & $0.21 \pm 0.08$ & $0.68 \pm 0.08 * *$ & 201 \\
\hline Abl-346 & gi33086496 & 88396 & 6.77 & $0.04 \pm 0.02$ & $0.05 \pm 0.01$ & $0.05 \pm 0.01$ & $0.01 \pm 0.00^{*}$ & 131 \\
\hline \multicolumn{9}{|l|}{ Inguinal WAT } \\
\hline Glutamic pyruvic transaminase 1 (GPT 1 ) & gi149066073 & 50476 & 6.63 & $0.19 \pm 0.03$ & $0.16 \pm 0.07$ & $0.24 \pm 0.03$ & $0.12 \pm 0.00^{*}$ & 62 \\
\hline Glutamine synthetase (GS) & gi228136 & 41153 & 6.38 & $0.06 \pm 0.03$ & $0.06 \pm 0.04$ & $0.12 \pm 0.02$ & $0.03 \pm 0.00^{*}$ & 74 \\
\hline Elongation factor 2 & gi8393296 & 96192 & 6.41 & $0.06 \pm 0.01$ & $0.07 \pm 0.01$ & $0.06 \pm 0.01$ & $0.02 \pm 0.00^{*}$ & 153 \\
\hline Proteosome subunit beta type 10 & gi71043724 & 24305 & 6.18 & $0.06 \pm 0.00$ & $0.07 \pm 0.01$ & $0.03 \pm 0.01$ & $0.10 \pm 0.01^{* *}$ & 66 \\
\hline Rat annexin V & gi 157836327 & 35458 & 4.97 & $0.45 \pm 0.13$ & $0.62 \pm 0.18$ & $0.29 \pm 0.13$ & $0.63 \pm 0.03 *$ & 218 \\
\hline Inosine phosphorylase & gi205829287 & 32566 & 6.46 & $0.41 \pm 0.05$ & $0.44 \pm 0.09$ & $0.28 \pm 0.06$ & $0.60 \pm 0.06^{* *}$ & 140 \\
\hline \multicolumn{9}{|l|}{ Abdominal WAT } \\
\hline Cytosolic non specific dipeptidase & gi58219062 & 53116 & 5.43 & $0.15 \pm 0.09$ & $0.12 \pm 0.02$ & $0.11 \pm 0.00$ & $0.14 \pm 0.01^{*}$ & 163 \\
\hline Septin 2 & gil 49037492 & 45403 & 6.10 & $0.01 \pm 0.00$ & $0.04 \pm 0.01$ & $0.15 \pm 0.05$ & $0.02 \pm 0.00^{*}$ & 70 \\
\hline Heat shock protein 90 (HSP90) & gi40556608 & 83571 & 4.97 & $0.04 \pm 0.02$ & $0.04 \pm 0.04$ & $0.11 \pm 0.02$ & $0.03 \pm 0.01 *$ & 116 \\
\hline Hemopexin & gil 22065203 & 52060 & 7.58 & $0.12 \pm 0.03$ & $0.34 \pm 0.14$ & $0.32 \pm 0.05$ & $0.14 \pm 0.02 *$ & 172 \\
\hline S glutathiolated carbonic anhydrase I & gi 157875870 & 29607 & 6.74 & $0.13 \pm 0.07$ & $0.05 \pm 0.01$ & $0.15 \pm 0.04$ & $0.04 \pm 0.03 *$ & 65 \\
\hline Glutathione $\mathrm{S}$ transferase & gi204499 & 22042 & 7.82 & $0.14 \pm 0.05$ & $0.19 \pm 0.09$ & $0.47 \pm 0.06$ & $0.12 \pm 0.03^{*}$ & 67 \\
\hline $\begin{array}{l}\text { Nicotinamide phosphoribosyltransferase } \\
\text { (NAMPT) }\end{array}$ & gi29293813 & 55689 & 6.69 & $0.10 \pm 0.03$ & $0.08 \pm 0.01$ & $0.07 \pm 0.02$ & $0.19 \pm 0.02 * *$ & 104 \\
\hline Catalase & gi6978607 & 60062 & 7.07 & $0.21 \pm 0.03$ & $0.15 \pm 0.04$ & $0.11 \pm 0.00$ & $0.20 \pm 0.01 * *$ & 98 \\
\hline NADP dependent malic enzyme & gi158341689 & 64545 & 6.35 & $0.42 \pm 0.07$ & $0.30 \pm 0.08$ & $0.17 \pm 0.04$ & $0.32 \pm 0.03 * *$ & 194 \\
\hline Pyruvate carboxylase & gi3 1543464 & 130436 & 6.34 & $0.01 \pm 0.00$ & $0.08 \pm 0.03$ & $0.01 \pm 0.00$ & $0.02 \pm 0.00 * *$ & 488 \\
\hline 2 oxoglutarate dehydrogenase & gi62945278 & 117419 & 6.30 & $0.12 \pm 0.02$ & $0.14 \pm 0.05$ & $0.16 \pm 0.00$ & $0.13 \pm 0.00^{* *}$ & 173 \\
\hline Glutamic pyruvic transaminase 1 (GPT 1 ) & gil 49066073 & 50476 & 6.63 & $0.07 \pm 0.02$ & $0.08 \pm 0.01$ & $0.07 \pm 0.01$ & $0.02 \pm 0.01 *$ & 97 \\
\hline Aspartyl t RNA synthase & gi16758642 & 57546 & 6.02 & $0.05 \pm 0.00$ & $0.04 \pm 0.00$ & $0.04 \pm 0.01$ & $0.15 \pm 0.02 * *$ & 75 \\
\hline Alpha $\mathbf{I}$ antitrypsin precursor & gi203063 & 445978 & 5.70 & $0.92 \pm 0.38$ & $0.54 \pm 0.02$ & $0.64 \pm 0.07$ & $0.45 \pm 0.07^{*}$ & 152 \\
\hline $\begin{array}{l}\text { G protein regulated inducer of neurite } \\
\text { outgrowth }\end{array}$ & gi 84000009 & 96065 & 8.14 & $0.34 \pm 0.11$ & $0.49 \pm 0.03$ & $0.41 \pm 0.04$ & $0.31 \pm 0.00^{*}$ & 71 \\
\hline
\end{tabular}

continued

pattern viz. gender different in both ND and HFD rats with higher protein abundances in males (Group I), gender different in both ND and HFD rats with higher protein abundances in females (Group II), gender different in both ND and HFD rats with opposite protein abundances between the genders (Group III), gender different only in HFD rats (Group IV), and gender different only in ND rats (Group V).

\section{Subcutaneous WAT}

As listed in Table 2, for subcutaneous WAT, 3 proteins were categorized into Group I, including zero beta globulin, desmin (DES), and serine/cysteine peptidase inhibitor. A total of 7 proteins were included in Group II (e.g. ezrin, NADH dehydrogenase, aflatoxin B1 aldehyde reductase, annexin $\mathrm{A} 1$, beta enolase, malate dehydrogenase, and creatine kinase $M$ type). Interestingly, an actin related

Mukherjee/Choi/Choi/Oh/Liu/Yun 


\begin{tabular}{|c|c|c|c|c|c|c|c|c|}
\hline \multirow[b]{2}{*}{ Protein } & \multirow[b]{2}{*}{ Acc. No. ${ }^{\prime \prime}$} & \multirow[b]{2}{*}{$\begin{array}{l}\text { Nominal mass } \\
(\mathrm{Mr})^{2}\end{array}$} & \multirow[b]{2}{*}{$\begin{array}{l}\text { Calculated } \\
\text { pl }\end{array}$} & \multicolumn{2}{|c|}{$\mathrm{ND}^{3)}$} & \multicolumn{2}{|c|}{$\mathrm{HFD}^{33}$} & \multirow[b]{2}{*}{ Score $^{5)}$} \\
\hline & & & & $\begin{array}{l}\text { Males }^{43} \\
(\% \text { vol })\end{array}$ & $\begin{array}{l}\text { Females } \\
\text { (\% vol) }\end{array}$ & $\begin{array}{l}\text { Males }^{4)} \\
(\% \text { vol) }\end{array}$ & $\begin{array}{l}\text { Females }{ }^{4)} \\
(\% \text { vol })\end{array}$ & \\
\hline \multicolumn{9}{|l|}{ Group V: Gender-different only in ND rats } \\
\hline \multicolumn{9}{|l|}{ Subcutaneous WAT } \\
\hline Serine / cysteine proteinase inhibitor & gil 49068858 & 45097 & 9.01 & $1.66 \pm 0.29$ & $0.67 \pm 0.41^{*}$ & $0.10 \pm 0.06$ & $0.55 \pm 0.30$ & 119 \\
\hline Aldose reductase & gi6978491 & 36230 & 6.26 & $0.07 \pm 0.01$ & $0.19 \pm 0.02 * *$ & $0.16 \pm 0.08$ & $0.12 \pm 0.04$ & 142 \\
\hline Proteosome 26S subunit & gil 49053606 & 56287 & 8.01 & $0.05 \pm 0.01$ & $0.17 \pm 0.01^{* *}$ & $0.05 \pm 0.02$ & $0.07 \pm 0.02$ & 163 \\
\hline Microtubule associated protein 2 kinase & gi5 15499 & 42456 & 6.16 & $0.16 \pm 0.05$ & $0.33 \pm 0.04$ * & $0.14 \pm 0.03$ & $0.11 \pm 0.02$ & 70 \\
\hline ER resident protein 29 precursor & gil 6758848 & 28614 & 6.23 & $0.02 \pm 0.01$ & $0.10 \pm 0.03^{*}$ & $0.13 \pm 0.08$ & $0.13 \pm 0.06$ & 81 \\
\hline Glycerol 3 phosphate dehydrogenase & gi57527919 & 38112 & 6.16 & $0.04 \pm 0.02$ & $0.14 \pm 0.03^{*}$ & $0.05 \pm 0.01$ & $0.06 \pm 0.00$ & 81 \\
\hline Inosine phosphorylase & gi205829287 & 32566 & 6.46 & $0.04 \pm 0.01$ & $0.13 \pm 0.02 *$ & $0.11 \pm 0.08$ & $0.15 \pm 0.01$ & 115 \\
\hline GDP -L-fucose synthase & gil 88536096 & 36059 & 6.26 & $0.05 \pm 0.02$ & $0.13 \pm 0.02 *$ & $0.04 \pm 0.01$ & $0.05 \pm 0.00$ & 126 \\
\hline \multicolumn{9}{|l|}{ Inguinal WAT } \\
\hline Aspartyl-tRNA synthase & gil 16758642 & 57546 & 6.02 & $0.05 \pm 0.01$ & $0.12 \pm 0.02 *$ & $0.09 \pm 0.01$ & $0.09 \pm 0.01$ & 90 \\
\hline Long chain specific acyl CoA dehydrogenase & gi6978431 & 48242 & 7.63 & $0.42 \pm 0.07$ & $0.19 \pm 0.02 *$ & $0.22 \pm 0.10$ & $0.17 \pm 0.01$ & 203 \\
\hline Pyruvate dehydrogenase & gi57657 & 43853 & 8.35 & $0.04 \pm 0.00$ & $0.11 \pm 0.00^{* *}$ & $0.13 \pm 0.00$ & $0.11 \pm 0.03$ & 91 \\
\hline Creatine kinase B type & gi31542401 & 42970 & 5.33 & $0.21 \pm 0.05$ & $0.44 \pm 0.06^{*}$ & $0.22 \pm 0.09$ & $0.22 \pm 0.04$ & 150 \\
\hline Aldose reductase & gi6978491 & 36230 & 6.26 & $0.33 \pm 0.03$ & $0.91 \pm 0.00^{* *}$ & $0.59 \pm 0.15$ & $0.62 \pm 0.19$ & 131 \\
\hline Isocitrate dehydrogenase $3\left(\mathrm{NAD}^{+}\right)$ & gil 49041704 & 39409 & 5.87 & $0.17 \pm 0.03$ & $0.34 \pm 0.07^{* *}$ & $0.24 \pm 0.09$ & $0.18 \pm 0.05$ & 87 \\
\hline Calreticulin precursor & gill 1693172 & 48137 & 4.33 & $0.42 \pm 0.02$ & $0.93 \pm 0.15^{*}$ & $0.37 \pm 0.06$ & $0.32 \pm 0.28$ & 199 \\
\hline Dismutase & gi818029 & 15740 & 5.89 & $1.16 \pm 0.37$ & $0.12 \pm 0.01^{*}$ & $0.22 \pm 0.15$ & $0.17 \pm 0.14$ & 111 \\
\hline \multicolumn{9}{|l|}{ Abdominal WAT } \\
\hline $\begin{array}{l}\text { Medium chain specific acyl CoA } \\
\text { dehydrogenase }\end{array}$ & gill 13018 & 46925 & 8.63 & $0.11 \pm 0.01$ & $0.40 \pm 0.09^{*}$ & $0.42 \pm 0.01$ & $0.29 \pm 0.01$ & 104 \\
\hline Hydroxysteroid dehydrogenase like protein 2 & gi7 1043858 & 58649 & 5.85 & $0.07 \pm 0.03$ & $0.17 \pm 0.01^{*}$ & $0.15 \pm 0.09$ & $0.03 \pm 0.00$ & 67 \\
\hline Peptidyl prolyl cis trans isomerase A & gi 8394009 & {$[809]$} & 8.34 & $0.10 \pm 0.04$ & $0.24 \pm 0.04^{*}$ & $0.01 \pm 0.00$ & $0.0 \mathrm{I} \pm 0.00$ & 82 \\
\hline Rat transthyretin & gi3212532 & 13122 & 6.04 & $0.63 \pm 0.13$ & $0.24 \pm 0.11^{*}$ & $0.10 \pm 0.03$ & $0.01 \pm 0.00$ & 114 \\
\hline Acetyl CoA acyltransferase 2 & gil 49027157 & 38753 & 8.64 & $0.57 \pm 0.04$ & $0.08 \pm 0.04 * *$ & $0.31 \pm 0.01$ & $0.27 \pm 0.08$ & 216 \\
\hline Periostin & gil 57823757 & 90683 & 7.27 & $0.08 \pm 0.01$ & $0.15 \pm 0.02 *$ & $0.09 \pm 0.04$ & $0.05 \pm 0.02$ & 87 \\
\hline Afamin isoform & gil 49033759 & 71135 & 5.87 & $0.07 \pm 0.01$ & $0.19 \pm 0.03^{*}$ & $0.19 \pm 0.06$ & $0.15 \pm 0.03$ & 66 \\
\hline 6 phosphogluconate dehydrogenase & gi2 293347815 & 53715 & 6.58 & $0.41 \pm 0.03$ & $0.32 \pm 0.02 *$ & $0.46 \pm 0.15$ & $0.57 \pm 0.03$ & 114 \\
\hline
\end{tabular}

Table 2. List of proteins showing gender-dependent expressions in three different white adipose tissues of lean and obese rats 1) NCBI nr database accession number. 2) The nominal mass is the integer mass of the most abundant naturally occurring stable isotope of an element. The nominal mass of a molecule is the sum of the nominal masses of the elements in its empirical formula. 3) Notations for experimental group division: ND, control rats fed a ND; HFD, rats fed a HFD. 4) Statistical significance was determined by ANOVA test, where $\mathrm{p}$-values is $* p<0.05$ and $* * p<0.01 . * * *$ indicates the $p$ value of each gender different protein in ND/ HFD or in both. 5) Protein scores greater than 61 are significant $(p<0.05)$.

protein 3 homolog showed gender-dependent expression but the protein abundances were the opposite between the genders (Group III). Among these protiens, DES and NADH dehydrogenase showed the highest gender differences in both ND and HFD rats. We also identified six proteins showing significant gender-dimorphism only in HFD rats (Group IV), and eight proteins were genderdependently expressed only in ND rats (Group V, Table 2).

\section{Inguinal WAT}

Group I proteins identified in inguinal WAT include hydroxyacyl CoA dehydrogenaase, fructose bisphosphate aldolase C, fatty acid synthase, rat mitochondrial 3,2
enoyl-CoA, S acyl fatty acid synthase thioesterase, NADH dehydrogenase (ubiquinine), ester hydrolase CII or f54 homolog, carbonic anhydrase 2. Group II contained proteins with various functions such as albumin, vinculin, protein disulfide isomerase associated 3 , mimecan, biliverdin reductase A. Group III included two proteins (e.g. cathepsin D and rat enoyl CoA hydratase), which have been reported with connection to obesity. Among these, S acyl fatty acid synthase thioesterase, biliverdin reductase $\mathrm{A}$ and rat enoyl CoA hydratase showed the most significant gender-different expression under both dietary conditions. In this deposit, we also detected several proteins showing gender-differences only in either ND (eight proteins) or HFD rats (six proteins) (Table 2). 


\section{Subcutaneous WAT}
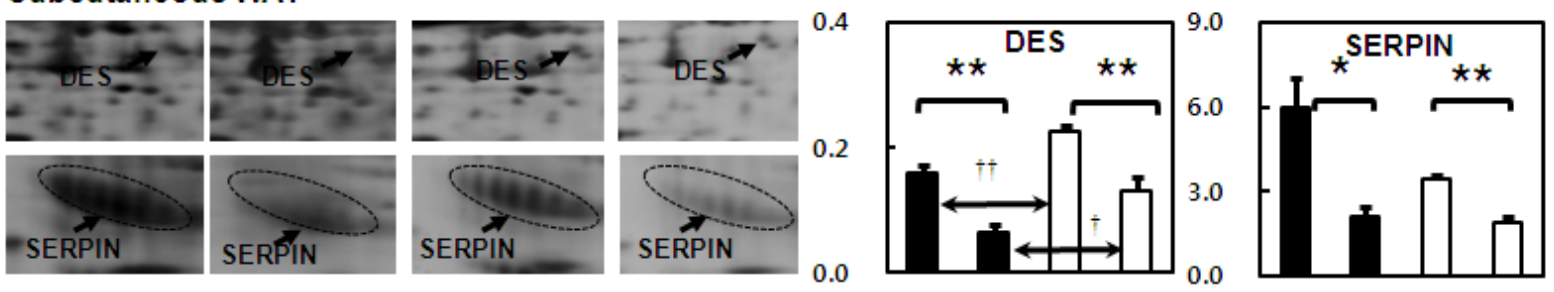

\section{Inguinal WAT}
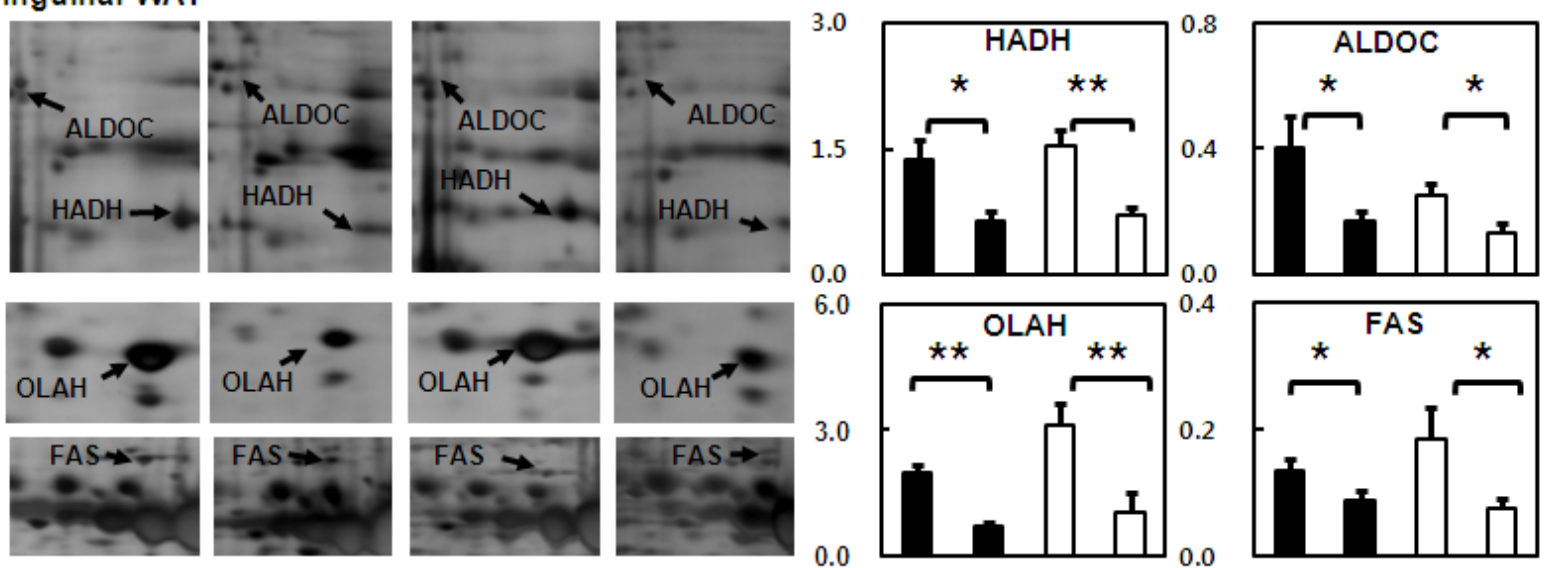

\section{Abdominal WAT}
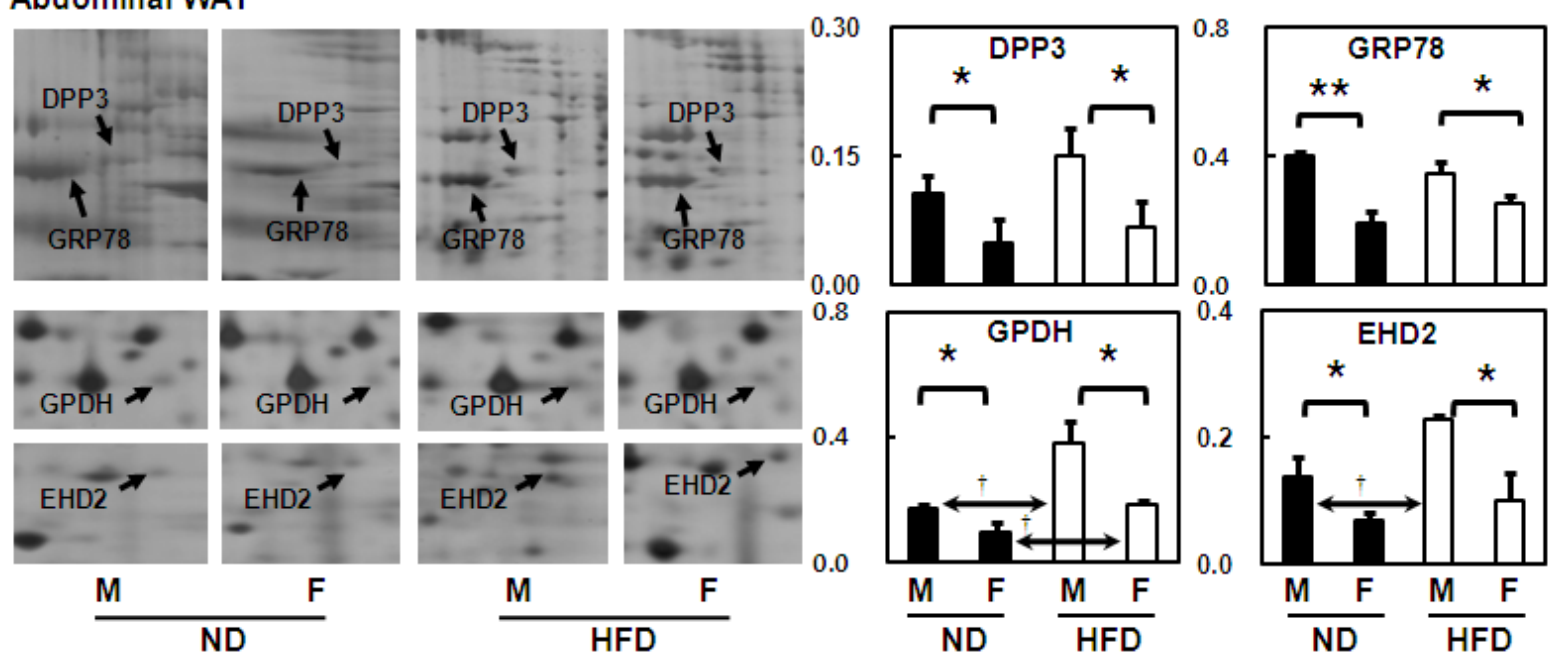

Fig. 2. Differentially expressed proteins showing gender differences in both ND and HFD rats with higher protein abundances in males. For the full name of each protein, see Table 2. Data are expressed as mean values \pm SD of the volume density (\%) of the changed spot in 3 individual gels using pooled WAT ( 3 types) from 7 rats per group. These proteins have $p$ values $<0.05$ when comparing males and females fed ND and/or HFD. Statistical significance between male (M) and female (F) rats as well as ND and HFD was determined by ANOVA test, where $p$ values were ${ }^{*} p<0.05$ and ${ }^{* *} p<0.01$ (between genders) and $\dagger p<0.05 ; \dagger \dagger p<0.01$ (between diet groups).

\section{Abdominal WAT}

The current proteomic analysis revealed that the highest number of proteins showing gender dimorphism occurred in abdominal WAT among the three deposits. A total of 48 proteins showed gender differences at least in ND or HFD rats. Group I contained some metabolically important proteins such as glycerol 3 phosphate dehydrogenase, NADH dehydrognase, and L-lactate dehydrogenase as well as other miscellaneous proteins including tpil protein, EH domain containing protein 2, aryl sulfotransferase, dipeptidyl peptidase 3 , cysteine sulfinate decarboxylase, and glucose regulated proteins.

Mukherjee/Choi/Choi/Oh/Liu/Yun 


\section{Subcutaneous WAT}
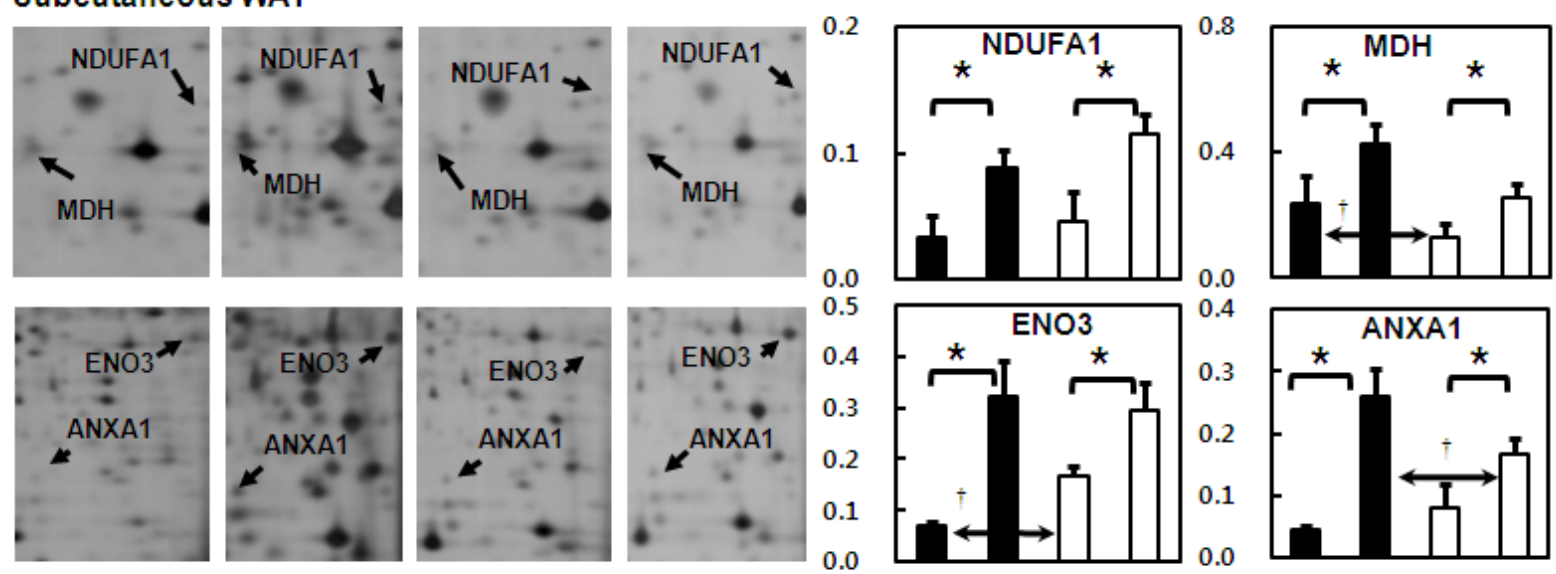

Inguinal WAT
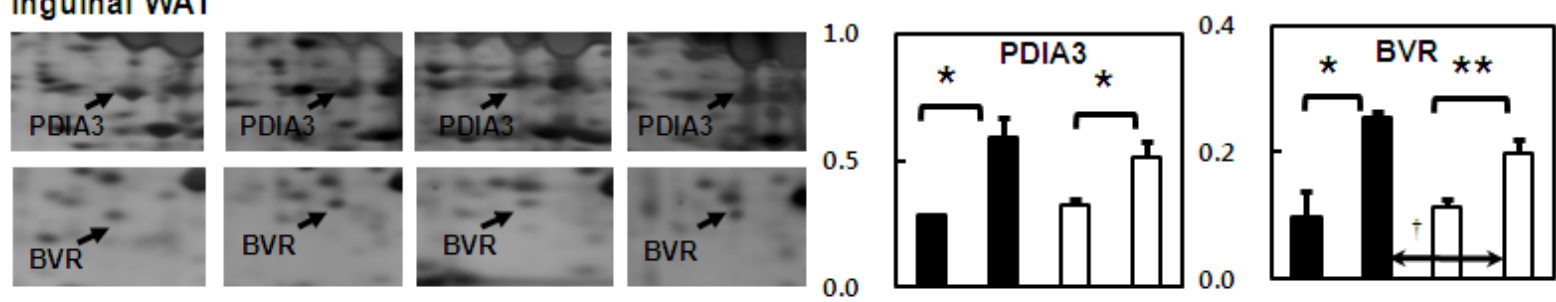

\section{Abdominal WAT}
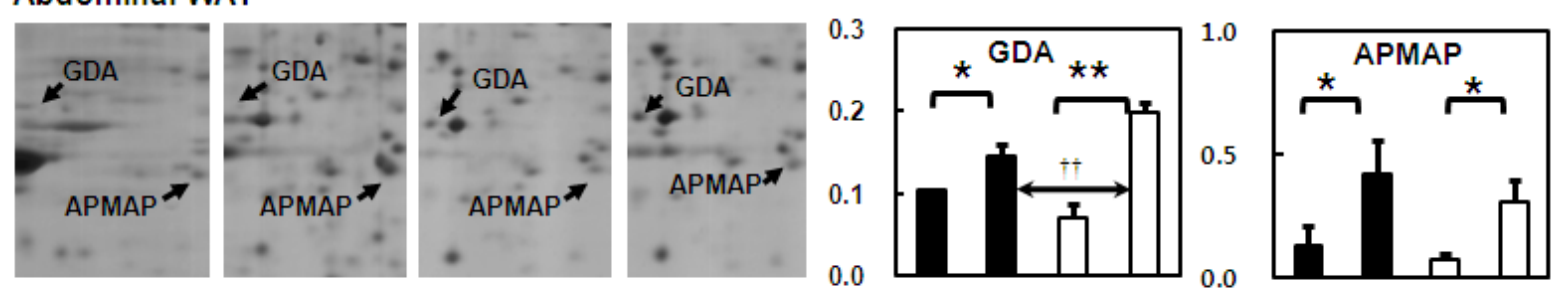

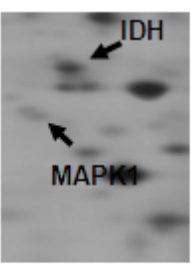

M

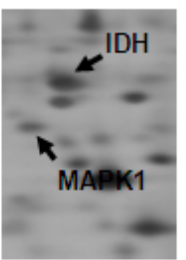

$\mathbf{F}$

ND
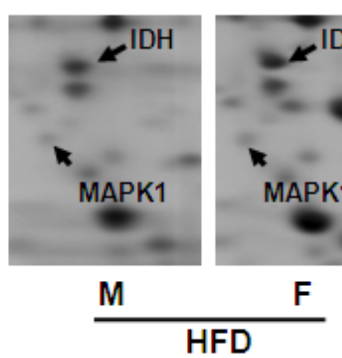
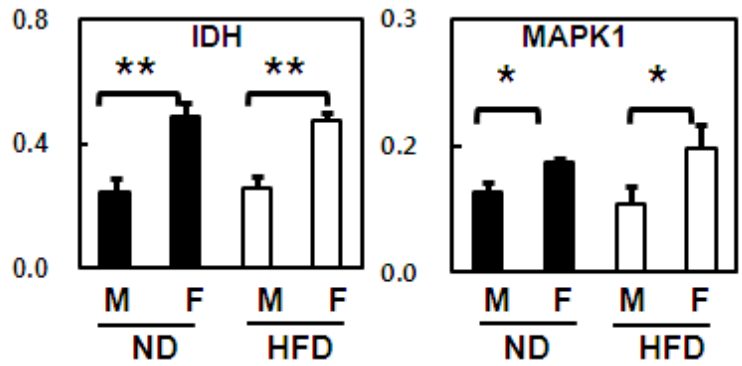

Fig. 3. Differentially expressed proteins showing gender differences in both ND and HFD rats with higher protein abundances in females. For the full name of each protein, see Table 2. Data are expressed as mean values \pm SD of volume density (\%) of the changed spot in 3 individual gels using pooled WAT ( 3 types) from 7 rats per group. These proteins have $p$ values $<0.05$ when comparing males and females fed ND and/or HFD. Statistical significance between male (M) and female (F) rats as well as ND and HFD was determined by ANOVA test, where $p$ values were ${ }^{*} p<0.05$ and ${ }^{* *} p<0.01$ (between genders) and $\dagger p<0.05 ; \dagger \dagger p<0.01$ (between diet groups).

Group II proteins included guanine deaminase, seryl aminoacyl tRNA synthase, macrophage capping protein, adipocyte plasma membrane associated protein, serpin B6, isocitrate dehydrogenase, mitogen activated protein kinase 1, Ca binding protein. Group III contained

Gender-dependent Protein Expression in White Adipose Tissues proteins important in respect of energy metabolism such as long chain fatty acid CoA ligase 1 , glycogen phosphorylase, creatine kinase $\mathrm{M}$ type, beta enolase, pyruvate kinase isozyme M1/M2, and moesin. Isocitrate dehydrogenase, glycerol 3 phosphate dehydrogenase 
Fig. 4. Differentially expressed proteins showing gender differences in both ND and HFD rats with opposite protein abundances between the genders. For the full name of each protein, see Table 2. Data are expressed as mean values $\pm \mathrm{SD}$ of volume density $(\%)$ of the changed spot in 3 individual gels using pooled WAT (3 types) from 7 rats per group. These proteins have $p$ values $<0.05$ when comparing males and females fed ND and/or HFD. Statistical significance between male $(\mathrm{M})$ and female $(\mathrm{F})$ rats as well as ND and HFD was determined by ANOVA test, where $p$ values were ${ }^{*} p<0.05$ and ${ }^{* *} p<0.01 \quad$ (between genders) and $\dagger p<0.05 ; \dagger \dagger$ $p<0.01$ (between diet groups).
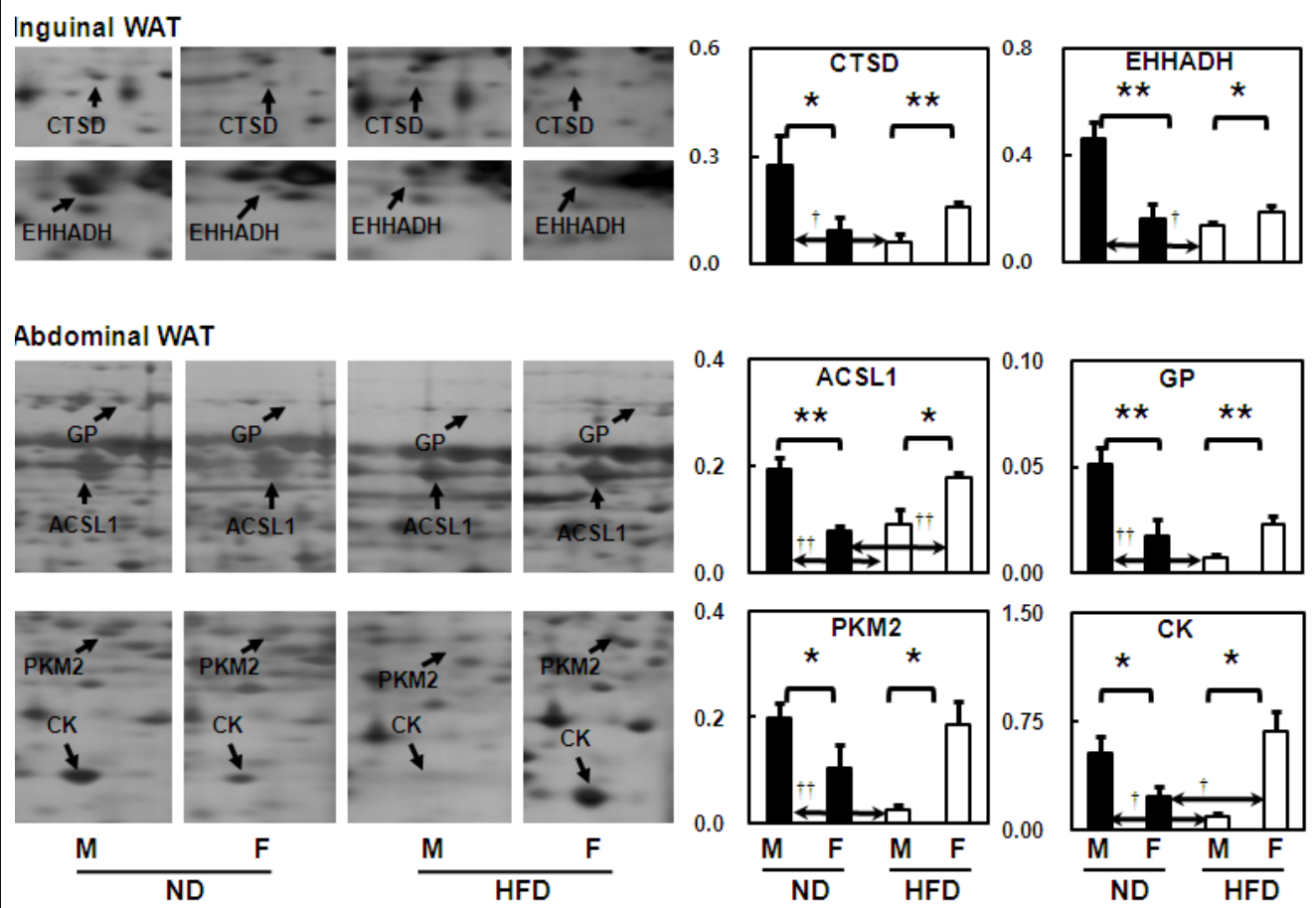

Fig. 5. Differentially expressed proteins showing gender differences only in HFD rats. For the full name of each protein, see Table 2. Data are expressed as mean values \pm SD of volume density (\%) of the changed spot in 3 individual gels using pooled WAT (3 types) from 7 rats per group. These proteins have $p$ values $<0.05$ when comparing males and females fed ND and/or HFD. Statistical significance between male $(\mathrm{M})$ and female $(\mathrm{F})$ rats as well as ND and HFD was determined by ANOVA test, where $p$ values were ${ }^{*} p<0.05$ and ${ }^{* *} p<0.01 \quad$ (between genders) and $\uparrow p<0.05 ; \dagger \dagger$ $p<0.01$ (between diet groups).
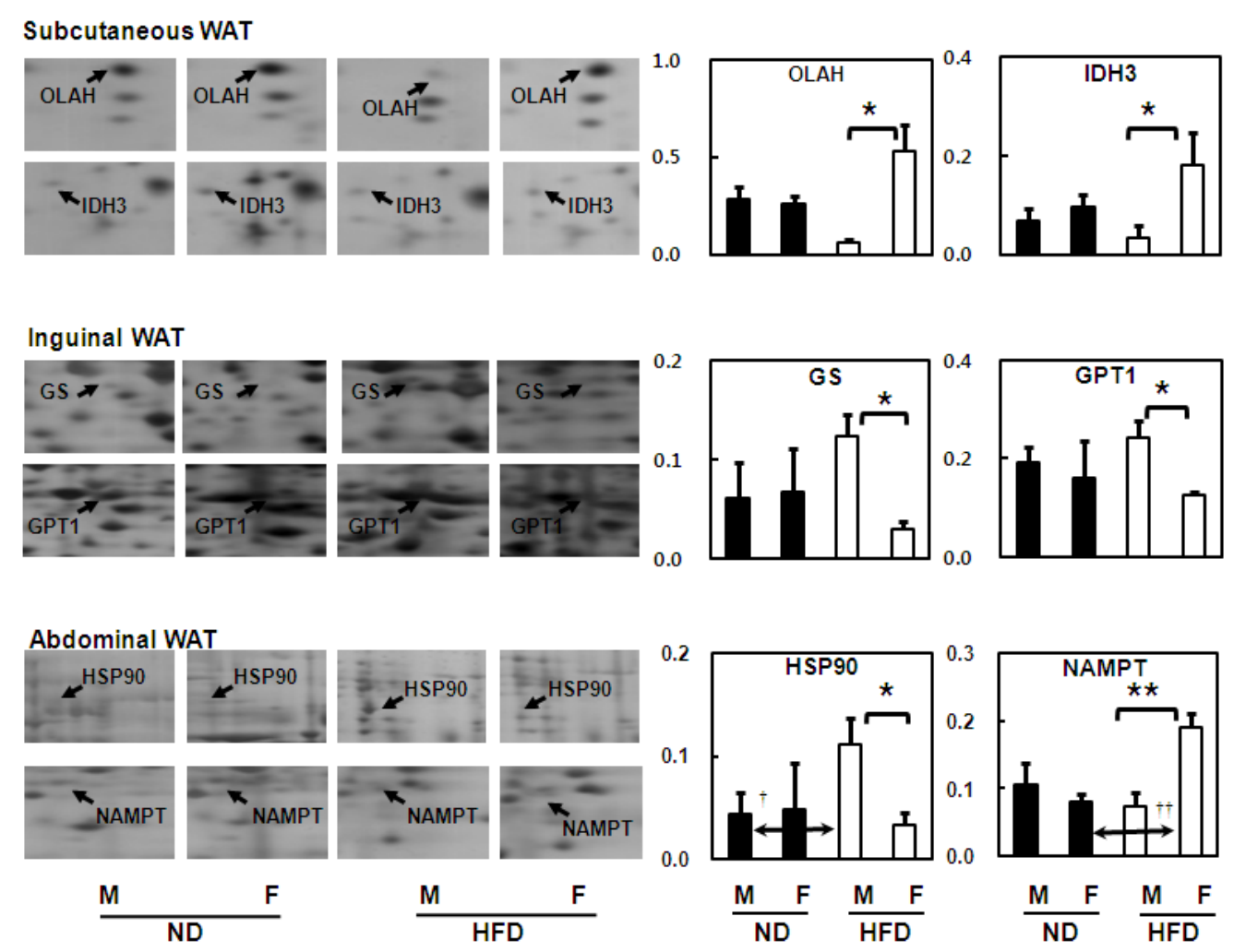

and glycogen phosphorylase exhibited the most significant gender-specific differential expression of each three group, respectively. Apart from these, the total number of proteins showing gender differences only in either ND or HFD rats was determined to be 8 and 15 , respectively, in abdominal WAT.

Mukherjee/Choi/Choi/Oh/Liu/Yun 
Fig. 6. Validation of proteomic data using immunoblot analysis. Levels of five proteins identified from 2-DE analysis were established using the pooled WAT (3 types) samples from 7 rats per group. For the full name of each protein, see Abbreviations. Data are representative of three independent experiments. Statistical significance between male (M) and female (F) rats as well as ND and HFD was determined by ANOVA test, where $p$ values were ${ }^{*} p<0.05$ and ${ }^{* *} p<0.01$ (between genders) and $\uparrow p<0.05 ; \uparrow \uparrow p<0.01$ (between diet groups).

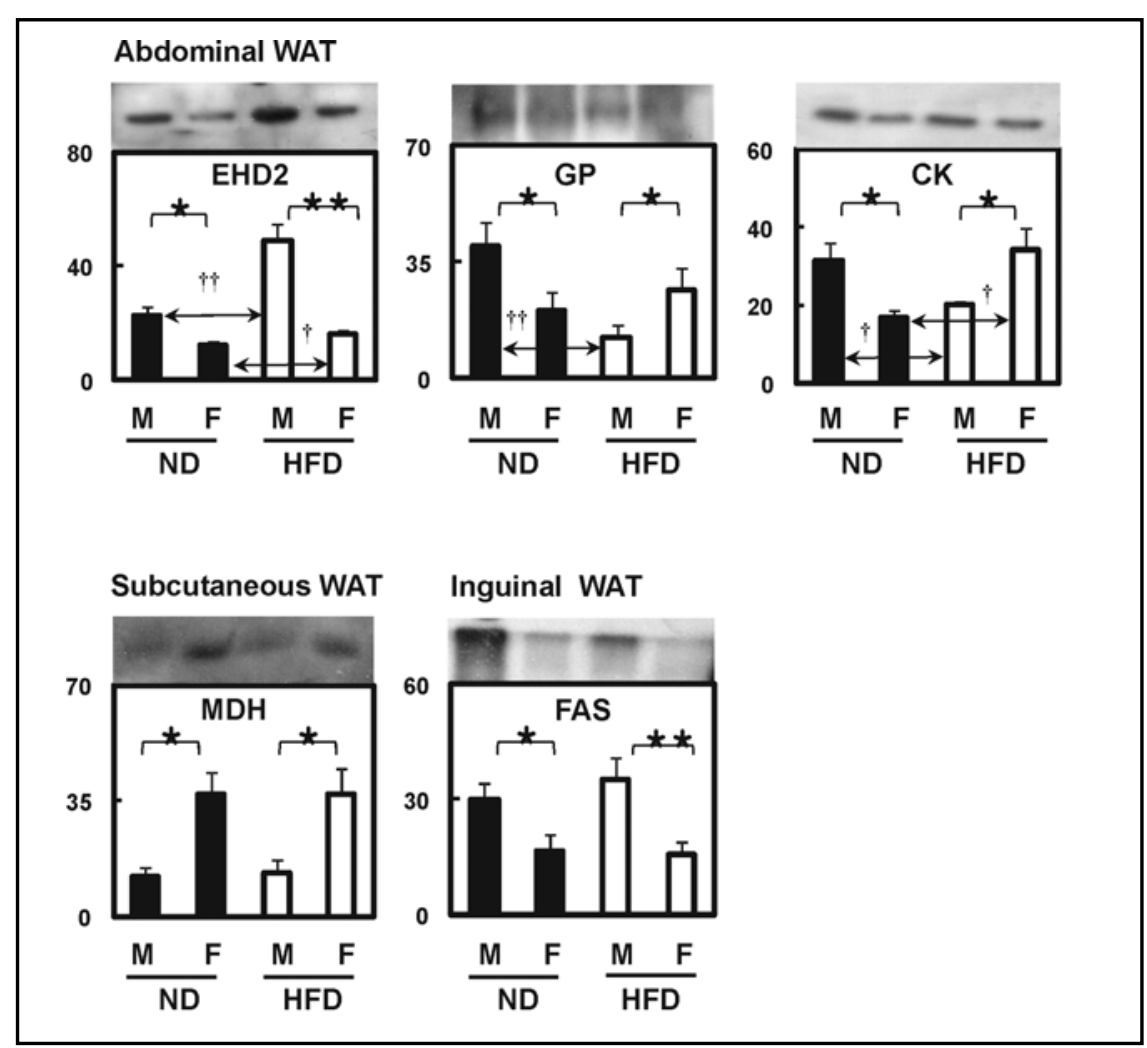

Validation of 2-DE data by immunoblot analysis

To varify the 2-DE proteomic data, the abundances of five proteins were further confirmed by immunoblot analysis. The protein levels of the three proteins of interest in abdominal WAT (e.g. EDH2, GP, and CK) and malate dehydrogenase (MDH) in subcutaneous WAT as well as FAS (fatty acid synthase) in inguinal WAT were confirmed. As shown in Fig. 6, the protein abundances of all examined proteins were in agreement with the data obtained from 2-DE analysis, demonstrating successful validation of our proteomic data.

\section{Discussion}

In the current proteomic study of three different WAT, we identified numerous proteins showing gender-dependent expression after 8 weeks breeding period. For many of these proteins, this was the first study to demonstrate a connection with obesity. To more easily interpret the proteomic data, we categorized these proteins into five groups as illustrated in Table 2. Due to space limitation, several representative proteins showing the most significant gender differences in each group but excluding group V (included proteins which are gender-dependently expressed only in ND rats) were discussed.

Gender-dependent Protein Expression in White Adipose Tissues
Proteins showing gender differences with higher abundances in both ND and HFD male rats (Group I)

In subcutaneous WAT, one of the most important findings was gender-specific differential expression of DES and serine/cysteine peptidase inhibitor (SERPIN). We found a significantly higher amount of DES in males both in ND and HFD rats (Fig. 2). DES is an intermediate filament protein and one of the major glomerular injury markers $[25,26]$. A recent study demonstrated that during diet-induced obesity, more ceramide as well as other sphingolipids were produced as a result glomerular injury, and DES levels were elevated in HFD-induced obese models [27]. Our current data showed that both genders have elevated levels of DES in HFD rats, but this effect was more pronounced one in males, which was correlated with their higher weight gain. This is the first report of gender-different regulation of DES in HFD-induced obesity.

Our data showed higher concentration of SERPIN in males for both diets. SERPIN or plasminogen activator inhibitor (PAI) is a member of serpin family and is responsible for inhibiting fibrinolysis [28]. Griffiths et al. [29] reported that adipose tissue secretes fibrinolytic proteins including PAI, and during weight loss by the use of metformin or thiazoladinediones, plasma PAI levels were reduced. Collectively, a higher concentration of PAI 
may lead to elevated inhibition of fibrinolysis in males (Fig. 2), which may result in a higher risk of cardiovascular disease in males than females.

In the case of inguinal WAT, the most important finding was the detection of higher concentrations of two lipogenic enzymes, FAS and S acyl fatty acid synthase thioesterase (OLAH), in males (Fig. 2). Both enzymes are actually part of an enzyme complex involved in fat synthesis $[30,31]$. Therefore, our proteomic data is likely to be associated with the fact that males are more susceptible to lipogenesis and ultimately obesity when expose to HFD.

Another important observation in this proteomic study was that higher protein abundances of mitochondrial hydroxyacyl CoA dehydrogenase $(\mathrm{HADH})$ were observed in males for both ND and HFD (Fig. 2). HADH is a well established mitochondrial glucose metabolizing enzyme in skeletal muscle [32]. Its higher concentration indicates greater consumption of glucose as an energy fuel, and is probably related to more fat deposition in males. According to a report by Bouwmann et al. [33], this enzyme may be related to higher fatty acid oxidation to increase plasma FFA concentration in males. Both our proteomic and biochemical data support this fact that males have a higher chance to develop insulin resistance as well as obesity. This is the first report demonstrating a possible role of HADH in adipose tissue and gender-different expression in relation to obesity.

Another interesting finding was the observation that males have higher protein levels of fructose bisphosphate aldolase C (ALDOC), which is a major glycolytic enzyme. Importantly, it also mediates the association of $\mathrm{F}$ actin with GLUT (glucose transporter) 4 [34] (Fig. 2). According to Bouwmann et al. [33], stimulation of ALDOC by insulin or substrate fructose bisphostphate or glyceraldehydes3-phosphate mediates higher expression of GLUT4 in the membrane and thus promotes GLUT4-mediated glucose uptake for increased triglyceride synthesis. Here, the reduced protein abundance of ALDOC in females when exposed to HFD-fed rats was believed to be associated with lower weight gain through reduced fat synthesis as well as a higher reliance on lipid as an energy fuel.

In abdominal WAT, the most pronounced gender difference in expression was observed for glycerol-3phosphate dehydrogenase (GPDH) (Fig. 2). It has already been reported that in human morbid obesity cases, the expression of this enzyme is at least 2-fold higher than the lean counterparts $[35,36]$. GPDH is the sole enzyme for the production of glycerol-3-phosphate, which is required for the production of ample triacylglycerol (TAG) in obesity. In addition, GPDH-knockout mouse also become less obese even when feed HFD. In the current study, males were shown to have higher amounts of GPDH than females under both dietary conditions, which was greater in HFD-fed rats. This may explain the higher adipose tissue mass in males.

Another important finding in the current proteomic study was the higher levels of dipeptidyl peptidase 3 (DPP3) in males fed both diets (Fig. 2). This enzyme has been shown to cleave hormones and chemokines in vitro. A previous in vivo study also showed that DPP cleaves glucagon family peptide and glucose-dependent insulinotropic polypeptide [37]. A recent report by Conarello et al. [38] indicated that DDP4-null mice were resistant to HFD-induced obesity and insulin resistance. Our proteomic data support that high protein level of DPP3 is part of obese state as it was highly expressed in abdominal WAT of HFD male rats. Taken together, we believe that DPPs are important factors for controlling obesity and may play a role in energy homeostasis and food intake.

Similarly, the EH domain containing protein 2 (EDH2) was also expressed in higher concentrations in males compared to females (Fig. 2). EHD was reported to be expressed in higher levels at the time of adipocyte differentiation or lipid accumulation in 3T3-L1 adipose cells. Also, this membrane protein plays an important role in insulin-mediated glucose uptake [39]. In our proteomic data, males had a greater amount of $\mathrm{EH}$ domain containing protein2 (EHD2), implying increased amount of insulinstimulated glucose uptake than females, which facilitates increased fatty acid synthesis and ultimately higher weight gain.

Our proteomic analysis of inguinal WAT also revealed elevated level of glucose regulated protein 78 (GRP78), which is a well known ER stress protein or chaperon protein in males (Fig. 2). It has been shown that adipose tissue of obese mice faces severe hypoxic condition as a result GRP along with other ER stress proteins were elevated [40-42]. Here, higher protein levels of GRP78 in males also reflects a severe obese state in males when expose to HFD.

Proteins showing gender differences with higher abundances in both ND and HFD female rats (Group II)

In subcutaneous WAT, the most interesting result was the higher concentration of annexin A1 (ANXA1) in females. It has been reported that expression of annexin A1 decreases with an increase in differentiation and lipid

Mukherjee/Choi/Choi/Oh/Liu/Yun 
accumulation in 3T3-L1 adipocytes [43]. Annexin A1knockout mice were reported to be resistant to the antiinflammatory function of glucocorticoids as well as produce elevated level of IL6 and fat mass [44]. In the current proteomic study, females had a higher amount of annexin A1 (Fig. 3), which may be related to the reduced fat mass in both ND and HFD rats.

Our proteomic data also revealed the presence of higher levels of mitochondrial NADH dehydrogenase and malate dehydrogenase in females (Fig. 3), which may be associated with elevated mitochondrial function in glucose metabolism, or it may result from the higher rate of beta oxidation in females $[45,46]$. In addition, MDH helps in gluconeogenesis or conversion of glycerol to glucose; thus, the higher MDH concentration in females may ultimately result in reduced a source of glycerol for fatty acid synthesis.

Beta-enolase, an important glycolytic enzyme, also showed higher abundances in females (Fig. 3), implying a higher rate of phosphoenolpyruvate production, a source of pyruvate and ultimately to acetyl Co A, which can be used in beta-oxidation. An earlier study showed that enolase activity was increased when niacin-bound choronium was used to alleviate HFD-induced obesity [47]. Similarly, our proteomic data revealed that the lower concentrations of beta-enolase in males were observed in WAT of male rats fed a HFD.

Proteomic data of inguinal WAT showed higher concentration of biliverdin reductase A (BVR) in females under both dietary conditions (Fig. 3). BVR is best known for its antioxidant activity but recently it was shown to play an important role in glucose metabolism as well as insulin resistance [48]. It can also minimize the effect of insulin resistance by taking part in the upstream signaling pathways as an upstream activator of insulin growth factor and MAPK [49]. Our proteomic data demonstrated that females have greater abundances of this protein, which may enhance glucose metabolism and make females more resistant to insulin resistance.

Another important finding in this fat deposit was the higher concentration of protein disulfide isomerase associated 3 (PDIA3) in females (Fig. 3). It is well established that PDIA3 is a part of the microsomal triglyceride transfer protein (MTP) [50, 51]. Whenever protein disulfide isomerase was separated from MTP, it lost its catalytic activity to transfer to low density lipoproteins [52]. It was reported that when PPAR (peroxisome proliferator-activated receptor) $\alpha$ expression increased, MTP gene expression also increased [53]. Thus, MTP may have some function in increasing the rate of fatty acid oxidation, which ultimately resulted in greater weight loss in females than males. Our proteomic data firmly demonstrated for the first time that there were reduced levels of PDIA3 in WAT, although its physiological role is still unknown.

In abdominal WAT, one of the most interesting proteins showing significant gender-differences in expression was guanine deaminase (GDA), which is involved in deamination of guanine to purine metabolism. An earlier proteomic study found that the protein abundances of GDA were decreased in HFD-fed obese rats and its levels increased again upon treatment with anti-obesity agent tungstate [18]. Although the physiological roles of GDA in obesity has not yet been defined, it can be hypothesized that its downregulation in obese rats may be associated with repression of the RhoGTPases signaling pathway, which would provide more guanine for the synthesis of guanosine triphosphate (GTP) [18].

Of particular interest, higher levels of the adipocyte plasma membrane-associated protein (APMAP) were detected in females for both ND and HFD. Although the precise biological function of APMAP is unknown at present, it is considered a marker of adipocyte differentiation since it is present mostly in mature adipocytes [54]. The higher protein abundances of APMAP in HFD female rats (Fig. 3) were reported in BAT in our previous proteomic study. Taken together, APMAP might play a pivotal role in facilitating lipid metabolism when exposed to HFD in females, but not in males.

Mitogen activated protein kinase 1 (MAPK1), a major signaling factor in many cellular events, also showed gender-different variations. Due to the fact that MAPK can efficiently phosphprylate PPAR $\gamma$, it has been demonstrated that the non-phosphorylated PPAR $\gamma$ is more lipogenic [55]. Collectively, higher concentration of MAPK1 in females may have contributed to MAPK1dependent PPAR $\gamma$ inactivation to affect the lipogenic capacity in females.

Proteins showing gender differences but opposite pattern in both ND and HFD rats (Group III)

Interestingly, we identified several proteins showing gender-dependent differential expression in both diet types, but exhibited opposite patterns between the genders (Group III) (Fig. 4). One of the differentially expressed proteins in the inguinal WAT group was rat enoyl CoA hydratase (EHHADH). This is one of the major lipid metabolic enzymes that facilitate the second step of beta- 
oxidation [56]. Current proteomic data demonstrated that males had lower abundances of this protein when given HFD. This result suggests that the adaptive capability to utilize fats overloaded in WAT was greater in females, which would further contribute to reduced weight gain by preventing lipid accumulation through the increased rate of beta-oxidation.

Another important protein showing opposite gender differences between the diets was cathepsin D (CTSD), an aspartic protease, which has been shown to degrade insulin and its receptors in liver [57]. This is the first report on gender-different expression of CTSD in adipose tissue of rat. In ND-fed rats, higher protein levels in males may be correlated with lower rate of insulin-stimulated glucose uptake. In contrast, the lower protein abundances of CTSD in females may reflect a higher rate of insulindependent glucose uptake. However, unexpectedly, this pattern was reversed when exposed to HFD. We were unable to interpret this result and thus the physiological significance of this change remains questionable.

In abdominal WAT, more proteins showed the opposite pattern of gender different expression between the ND and HFD rats. The first example is the long chain fatty acid CoA ligase 1 (ACSL1), which is responsible for the conversion of fatty acids to fatty acyl CoAs for their oxidation in mitochondria $[31,58]$. When exposed to HFD, the protein levels of ACSL1 was greater in females by a factor of 2 when compared to ND female rats. This result suggests that adaptive capability to utilize fats overloaded in WAT was greater in females, which would contribute to reduced weight gain by preventing lipid accumulation through an increased rate of betaoxidation.

Another important glycolytic enzyme in this group worth discussing was pyruvate kinase M1/M2 (PKM1/ M2), which converts puruvate to phosphoenol pyruvate (PEP). Pyruvate can be used as an energy source in the glycolysis and citric acid cycle. It also acts as a mother molecule of acetyl CoA, which can be used for fatty acid biosynthesis [59]. Our data showed higher amounts of $\mathrm{PKM} 1 / \mathrm{M} 2$ in males than females in ND rats, which facilitates the partitioning of pyruvate to PEP for glycolysis. However, when fed a HFD, the protein levels of PKM1/ M2 in males was reduced by a factor of 5, implying reduced capability for the conversion of pyruvate to PEP through glycolysis, and ultimately more accumulated pyruvate for production of acetyl CoA for fatty acid synthesis. Conversely, females had a higher concentration of PKM1/M2 when fed a HFD, suggesting a higher rate of consumption of pyruvate through the glycolytic pathway, which would result in lower concentration of pyruvate for use in fatty acid synthesis.

Similar gender-different regulation was also observed for glycogen phopshorylase (GP), which is a major enzyme for glycogenolysis. This protein has also been reported to be controlled by insulin in adipose tissues [60]. An earlier study also showed that transition from carbohydrate to fat was always followed by glycogen deposition in adipose tissue, thus we hypothesized that HFD male rats have a higher amount of glycogen in adipose tissue [61]. Our proteomic data demonstrated that males have a higher concentration of GP in ND rats than females, raising a possibility for a facilitated glycogen break down than females. However, this phenomenon was reverse when the rats were exposed to HFD, the GP concentrations were significantly reduced in males but not in females. To conclude, higher protein levels of GP rather than enzyme activity dose not directly reflect greater utilization of carbohydrates than fats as an energy fuel.

The higher level of creatine kinase (CK) only in HFD female rats not in ND rats is of significant interest. CK is a well-recognized protein involved in robust energy homeostasis by promoting the reversible conversion of creatine to phosphorcreatine at the expense of ATP [62]. Based on the differential protein levels of CK between the genders, we postulate that males have a greater capacity of energy dissipation than females under normal dietary condition, but females have a greater adaptive capability against high fat loading (in HFD rats). Furthermore, higher levels of CK in HFD female rats suggest a greater capacity for use of phosphocreatine as an energy buffer providing ATP for cytosolic reactions during uncoupling reactions in HFD-induced obese rats.

\section{Proteins showing gender differences only in HFD rats (Group IV)}

Proteomic analysis of the three types of WAT enabled us to detect several proteins that showed gender dimorphism only in HFD rats. Of these, OLAH, isocitrate dehydrogenase $3\left(\mathrm{NAD}^{+}\right)$(IDH3) of subcutaneous WAT and nicotinamide phosphoribosyltransferase (NAMPT) of abdominal WAT displayed higher abundances in females, whereas the protein levels of glutamine synthatase (GS), glutamic pyruvic transaminase (GPT1) of inguinal WAT and heat shock protein 90 (HSP90) of abdominal WAT were in higher amount in males.

Interestingly, gender-different variations in the protein levels of two glutamine metabolic enzymes were observed in inguinal WAT. Like in subcutaneous WAT,

Mukherjee/Choi/Choi/Oh/Liu/Yun 
GS was present in higher concentrations in males, which could be related to a higher chance of adipogenesis since this enzyme was reported to be overexpressed by more than a 100-fold during insulin-stimulated adipogenesis in 3T3-L1 adipocytes [63, 64]. Similarly, the levels of GPT1 were also higher in HFD male rats than their female counterparts. GPT1 is an extracellular matrix enzyme of adipose tissue and plays a major role in the glycerogenic pathway as well as in pyruvate formation in adipose tissues $[65,66]$. Based on these findings, a higher amount of pyruvate and glycerol in males may be associated with increased lipogenesis. Collectively, it can be hypothesized that both GS and GPT1 directly or indirectly affect the events of lipogenesis or adipogenesis in rat WAT.

When exposed to HFD, only male rats showed a significant increase in HSP90, which was more than 2fold higher in HFD rats than ND rats. HSP 90 is a member of the heat shock protein family, which is up-regulated in response to cellular stress $[67,68]$. Thus, the increase in this protein reflected the severe state of obesity in HFD male rats. In contrast, the protein abundances of isocitrate dehydrogenase 3 (IDH3) were higher in HFD female rats. IDH catalyzes the decarboxylation of isocitrate in the TCA cycle, and this enzyme is the rate-limiting enzyme for the oxidation of TCA cycle substrates $[69,70]$. This data supports our hypothesis that females exhibit higher mitochondrial metabolism.

In abdominal WAT, we found another interesting protein that displayed gender-different expression, NAMPT. This protein, which is also known as visfatin, is specifically secreted by visceral adipose tissue. Visfatin exerts insulin-mimicking effects, thereby decreasing blood glucose levels [71]. Our data showed higher concentration of visfatin in females when exposed to HFD, which may contribute to lower blood glucose levels as well as increased fat oxidation to balance the depletion of blood sugar.

Finally, it is worth noting that a different expression pattern of OLAH was observed between subcutaneous and inguinal WAT. As shown in Fig. 2, the OLAH levels in inguinal WAT were higher in males for both diets. However, its abundances in subcutaneous fat were the highest in female HFD rats without gender-differences in ND rats (Fig. 5). Considering OLAH's biological functions in lipogenesis [30,72], we postulate that OLAH may play an important role in lipogenesis only in subcutaneous fat deposit but not in other fat deposits. Further studies are required to directly test this hypothesis.

As discussed above, our proteomic data revealed that males have a higher amount of lipogenic enzymes

Gender-dependent Protein Expression in White Adipose Tissues compared to females. Some important lipogenic enzymes like FAS, OLAH, GS, GPDH were present in higher concentrations, irrespective of dietary groups and WAT tissue types. On the other hand, females had a higher concentration of numerous mitochondrial enzymes including NDUF1, MDH, IDH3 and PC, which may lead to a higher energy homeostasis or energy dissipation capability in females when compared to males. We believe that these differential protein abundances are closely related with the fact that males gain more weight in response to HFD than females.

Collectively, susceptibility to obesity development in rats fed an HFD may, at least in part, result from differences in the regulation of key lipogenic and $\beta$ oxidation enzymes. In this regard, we hypothesized that male rats undergo slower fat oxidation, thereby leading to excessive accumulation of dietary fat in white adipose tissues when compared with female rats. Similar genderdimorphism was observed in non-exercised mice muscle [73].

Our comparative proteomic analysis of adipose tissues points to the existence of sex-associated differences in the abundance of numerous proteins. We believe that gender-different protein regulation in adipose tissues resulted from hormonal and metabolic differences. For example, testosterone might induce an increase in leptin production, thereby increasing calorie intake. Estrogen might induce a reduction in adipose mass and adipocyte size, as well as down regulate lipogenic enzymes and shift the partition of FFA towards oxidation and away from TG storage by activation of AMPK (AMP-activated protein kinase) and PPAR. In conclusion, the present proteomic research into gender-dimorphic pathophysiological mechanisms would aid in improving gender awareness in the health care system and in the implementation of evidence-based gender specific clinical recommendations.

\section{Abbreviations}

2-DE (two dimensional electrophoresis); ALDOC (fructose bisphosphate aldolase C); APMAP (adipocyte plasma membrane-associated protein); CK (creatine kinase); CTSD (cathepsin D); DES (desmin); FAS (fatty acid synthase); FFA (free fatty acid); GDA (guanine deaminase); GLUT (glucose transporter); GP (glycogen phopshorylase); GPDH (glycerol-3-phosphate dehydrogenase); GPT1 (glutamic pyruvic transaminase); GS (glutamine synthatase); HADH (hydroxyacyl CoA 
dehydrogenase); HFD (high fat diet); IDH (isocitrate dehydrogenase); MDH (malate dehydrogenase); ND (normal diet); OLAH (S acyl fatty acid synthase thioesterase); PDIA3 (protein disulfide isomerase associated 3); PKM1/M2 (pyruvate kinase M1/M2); PPAR (peroxisome proliferator-activated receptor); SERPIN (serine/cysteine peptidase inhibitor); TG (triglyceride); WAT (white adipose tissue).

\section{Acknowledgements}

This research was supported by the Mid-career Researcher Program (grant number 2011-0000509) and SRC program (Center for Food \& Nutritional Genomics: grant number 2011-0000914) of the National Research Foundation of Korea (NRF) funded by the Ministry of Education, Science and Technology. Rajib Mukherjee, Jung-Won Choi, Duk Kwon Choi, Tae Seok Oh, Hao Liu received BK21 scholarship.

\section{References}

$\rightarrow 1$ Kopelman PG: Obesity as a medical $>12$ problem. Nature 2000;404:635-643.

2 Mokdad AH, Ford ES, Bowman BA, Dietz WH, Vinicor F, Bales VS, Marks JS: Prevalence of obesity, diabetes, and obesity-related health risk factors, 2001 J Clin Endocrinol Metab 2003;289:7679.

-3 Kershaw EE, Flier JS: Adipose tissue as an endocrine organ. J Clin Endocrinol Metab 2004;89:2548-2556.

$\checkmark 4$ Siiteri PK: Adipose tissue as a source of hormones. Am J Clin Nutr 1987;45:277282.

$\checkmark 5$ Vettor R, De Pergola G, Pagano C, Englaro P, Laudadio E, Giorgino F, Blum WF, Giorgino R, Federspil G: Gender differences in serum leptin in obese people: Relationships with testosterone, body fat distribution and insulin sensitivity. Eur J Clin Inves 16 1997;27:1016-1024.

-6 Power ML, Schulkin J, Rossouw JE: Evolving practice patterns and attitudes toward hormone therapy of obstetriciangynecologists. Menopause 2007;14:2028.

7 Blaak E: Gender differences in fat metabolism. Curr Opin Clin Nutr Metab Care 2001;4:499-502.

$\rightarrow 8$ Sweeting HN: Gendered dimensions of obesity in childhood and adolescence. Nutr J 2008; 7:1.

$>9$ Shi H, Clegg DJ: Sex differences in the regulation of body weight. Physiol Behav 2009;97:199-204.

10 Wisniewski AB, Chernausek SD: Gender in childhood obesity: Family environment, hormones, and genes. Gend Med 2009;6 Suppl 1:76-85.

11 Mittendorfer B: Sexual dimorphism in human lipid metabolism. J Nutr 2005;135:681-686.
Zillikens MC, Yazdanpanah M, Pardo 20 LM, Rivadeneira F, Aulchenko YS, Oostra BA, Uitterlinden AG, Pols HA, van Duijn CM: Sex-specific genetic effects influence variation in body composition. Diabetologia 2008;51:2233-2241.

13 Kautzky-Willer A, Handisurya A: Metabolic diseases and associated complications: Sex and gender matter! Eur J Clin Invest 2009;39:631-648.

Liu H, Choi JW, Yun JW: Gender differences in rat plasma proteome in response to high-fat diet. Proteomics 2012;12:269-283.

15 Oh TS, Choi JW, Choi DK, Mukherjee R, Liu H, Yun JW: Gender dimorphism in skeletal muscle proteome between lean and diet-induced obese rats. Cell Physiol Biochem 2011;28:981-996.

Choi DK, Oh TS, Choi JW, Mukherjee R, Wang X, Liu H, Yun JW: Gender difference in proteome of brown adipose tissues between male and female rats exposed to a high fat diet. Cell Physiol Biochem 2011;28:933-948.

17 Wang X, Choi JW, Oh TS, Choi DK, Mukherjee R, Liu H, Yun JW: Comparative hepatic proteome analysis between lean and obese rats fed a high fat diet reveals existence of gender differences. Proteomics 2012;12:284299.

18 Barcelo-Batllori S, Corominola H, Claret M, Canals I, Guinovart J, Gomis R: Target identification of the novel antiobesity agent tungstate in adipose tissue from obese rats. Proteomics 2005;5:49274935.

19 Chen X, Hess S: Adipose proteome $>26$ analysis: Focus on mediators of insulin resistance. Expert Rev Proteomics 2008;5:827-839.
Ortega FJ, Mayas D, Moreno-Navarrete JM, Catalan V, Gomez-Ambrosi J, Esteve E, Rodriguez-Hermosa JI, Ruiz B, Ricart W, Peral B, Fruhbeck G, Tinahones FJ, Fernandez-Real JM: The gene expression of the main lipogenic enzymes is downregulated in visceral adipose tissue of obese subjects. Obesity (Silver Spring) 2010;18:13-20.

$\$ 21$ Bradford MM: A rapid and sensitive method for the quantitation of microgram quantities of protein utilizing the principle of protein-dye binding. Anal Biochem 1976;72:248-254.

$\checkmark 22$ Wang X, Choi JW, Joo JI, Kim DH, Oh TS, Choi DK, Yun JW: Differential expression of liver proteins between obesity-prone and obesity-resistant rats in response to a high-fat diet. Br J Nutr 2011;106:612-626.

Joo JI, Kim DH, Choi JW, Yun JW: Proteomic analysis for antiobesity potential of capsaicin on white adipose tissue in rats fed with a high fat diet. J Proteome Res 2010;9:2977-2987.

24 Joo JI, Oh TS, Kim DH, Choi DK, Wang $\mathrm{X}$, Choi JW, Yun JW: Differential expression of adipose tissue proteins between obesity-susceptible and resistant rats fed a high-fat diet. Proteomics 2011;11:1429-1448.

Floege J, Alpers CE, Sage EH, Pritzl P, Gordon K, Johnson RJ, Couser WG: Markers of complement-dependent and complement-independent glomerular visceral epithelial cell injury in vivo. Expression of antiadhesive proteins and cytoskeletal changes. Lab Invest 1992;67:486-497.

Alpers CE, Hudkins KL, Floege J, Johnson RJ: Human renal cortical interstitial cells with some features of smooth muscle cells participate in tubulointerstitial and crescentic glomerular injury. J Am Soc Nephrol 1994;5:201-209. 
27 Boini KM, Zhang C, Xia M, Poklis JL, Li PL: Role of sphingolipid mediator ceramide in obesity and renal injury in mice fed a high-fat diet. J Pharmacol Exp Ther 2010;334:839-846.

-28 Binder BR, Christ G, Gruber F, Grubic N, Hufnagl P, Krebs M, Mihaly J, Prager GW: Plasminogen activator inhibitor 1 : Physiological and pathophysiological roles. News Physiol Sci 2002;17:56-61.

-29 Griffiths SL, Grainger DJ: Proposal of a novel diabetogenic mechanism involving the serpin pai-1. Bioessays 2006;28:629641 .

\30 Smith S, Agradi E, Libertini L, Dileepan $\mathrm{KN}$ : Specific release of the thioesterase component of the fatty acid synthetase multienzyme complex by limited trypsinization. Proc Natl Acad Sci U S A 1976;73:1184-1188.

-31 Smith S, Witkowski A, Joshi AK: Structural and functional organization of the animal fatty acid synthase. Prog Lipid Res 2003;42:289-317.

\2 Dourmashkin JT, Chang GQ, Gayles EC, Hill JO, Fried SK, Julien C, Leibowitz SF: Different forms of obesity as a function of diet composition. Int $\mathrm{J}$ Obes (Lond) 2005;29:1368-1378.

$\checkmark 33$ Bouwman FG, Claessens M, van Baak MA, Noben JP, Wang P, Saris WH, Mariman EC: The physiologic effects of caloric restriction are reflected in the in vivo adipocyte-enriched proteome of 45 overweight/obese subjects. J Proteome Res 2009;8:5532-5540.

$\checkmark 34$ Kao AW, Noda Y, Johnson JH, Pessin JE, Saltiel AR: Aldolase mediates the association of f-actin with the insulinresponsive glucose transporter glut4. J Biol Chem 1999;274:17742-17747.

$\checkmark 35$ Kozak LP, Kozak UC, Clarke GT: Abnormal brown and white fat development in transgenic mice overexpressing glycerol 3-phosphate dehydrogenase. Genes Dev 1991;5:22562264.

-36 Swierczynski J, Zabrocka L, Goyke E, Raczynska S, Adamonis W, Sledzinski Z: Enhanced glycerol 3-phosphate dehydrogenase activity in adipose tissue of obese humans. Mol Cell Biochem 2003;254:55-59.

$\$ 37$ Mest HJ, Mentlein R: Dipeptidyl 48 peptidase inhibitors as new drugs for the treatment of type 2 diabetes. Diabetologia 2005;48:616-620.

$\checkmark 38$ Conarello SL, Li Z, Ronan J, Roy RS, Zhu L, Jiang G, Liu F, Woods J, Zycband E, Moller DE, Thornberry NA, Zhang BB: Mice lacking dipeptidyl peptidase iv are protected against obesity and insulin resistance. Proc Natl Acad Sci U S A 2003;100:6825-6830.

$\checkmark 39$ Bluher M, Wilson-Fritch L, Leszyk J, Laustsen PG, Corvera S, Kahn CR: Role of insulin action and cell size on protein expression patterns in adipocytes. J Biol Chem 2004;279:31902-31909.
Nakatani Y, Kaneto H, Kawamori D, Yoshiuchi K, Hatazaki M, Matsuoka TA, Ozawa K, Ogawa S, Hori M, Yamasaki Y, Matsuhisa M: Involvement of endoplasmic reticulum stress in insulin resistance and diabetes. J Biol Chem 2005;280:847-851.

Hosogai N, Fukuhara A, Oshima K, Miyata Y, Tanaka S, Segawa K, Furukawa S, Tochino Y, Komuro R, Matsuda M, Shimomura I: Adipose tissue hypoxia in obesity and its impact on adipocytokine dysregulation. Diabetes 2007;56:901911.

-42 Ye R, Mareninova OA, Barron E, Wang M, Hinton DR, Pandol SJ, Lee AS: Grp78 heterozygosity regulates chaperone balance in exocrine pancreas with differential response to cerulein-induced acute pancreatitis. Am J Pathol 2010;177:2827-2836.

Wong WT, Frost SC, Nick HS: Proteinsynthesis-dependent induction of annexin i by glucocorticoid. Biochem $\mathrm{J}$ 1991;275:313-319.

Warne JP, John CD, Christian HC, Morris JF, Flower RJ, Sugden D, Solito E, Gillies GE, Buckingham JC: Gene deletion reveals roles for annexin a 1 in the regulation of lipolysis and il-6 release in epididymal adipose tissue. Am J Physiol Endocrinol Metab 2006;291:E12641273.

Johnson HS: Nadp-malate dehydrogenase: Photoactivation in leaves of plants with calvin cycle photosynthesis. Biochem Biophys Res Commun 1971;43:703-709.

$\checkmark 46$ Jove M, Salla J, Planavila A, Cabrero A, Michalik L, Wahli W, Laguna JC, Vazquez-Carrera M: Impaired expression of nadh dehydrogenase subunit 1 and ppargamma coactivator- 1 in skeletal muscle of zdf rats: Restoration by troglitazone. J Lipid Res 2004;45:113123.

47 Rink C, Roy S, Khanna S, Rink T, Bagchi D, Sen CK: Transcriptome of the subcutaneous adipose tissue in response to oral supplementation of type 2 leprdb obese diabetic mice with niacin-bound chromium. Physiol Genomics 2006;27:370-379.

Wu B, Liu X, Shen J: Old biliverdin reductase: Links to insulin resistance and may be a novel therapeutic target. Med Hypotheses 2008;71:73-76.

Li LF, Li J: Link between oxidative stress and insulin resistance. Chin Med Sci 2007;22:254-259.

$>50$ Lin MC, Gordon D, Wetterau JR: Microsomal triglyceride transfer protein (mtp) regulation in hepg2 cells: Insulin negatively regulates mtp gene expression. J Lipid Res 1995;36:1073-1081.
St-Pierre J, Lemieux I, Miller-Felix I, Prud'homme D, Bergeron J, Gaudet D, Nadeau A, Despres JP, Vohl MC: Visceral obesity and hyperinsulinemia modulate the impact of the microsomal triglyceride transfer protein $-493 \mathrm{~g} / \mathrm{t}$ polymorphism on plasma lipoprotein levels in men. Atherosclerosis 2002;160:317-324.

$>52$ Wetterau JR, Aggerbeck LP, Bouma ME, Eisenberg C, Munck A, Hermier M, Schmitz J, Gay G, Rader DJ, Gregg RE: Absence of microsomal triglyceride transfer protein in individuals with abetalipoproteinemia. Science 1992;258:999-1001.

$\$ 53$ Stefano JT, de Oliveira CP, CorreaGiannella ML, de Lima VM, de Sa SV, de Oliveira EP, de Mello ES, Giannella-Neto D, Alves VA, Carrilho FJ: Nonalcoholic steatohepatitis (nash) in ob/ob mice treated with yo jyo hen shi ko (yhk): Effects on peroxisome proliferatoractivated receptors (ppars) and microsomal triglyceride transfer protein (mtp). Dig Dis Sci 2007;52:3448-3454. Albrektsen T, Richter HE, Clausen JT, Fleckner J: Identification of a novel integral plasma membrane protein induced during adipocyte differentiation. Biochem J 2001;359:393-402.

$>55$ Adams M, Reginato MJ, Shao D, Lazar MA, Chatterjee VK: Transcriptional activation by peroxisome proliferatoractivated receptor gamma is inhibited by phosphorylation at a consensus mitogenactivated protein kinase site. J Biol Chem 1997;272:5128-5132.

Bahnson BJ, Anderson VE, Petsko GA: Structural mechanism of enoyl-coa hydratase: Three atoms from a single water are added in either an elcb stepwise or concerted fashion. Biochemistry 2002;41:2621-2629.

57 Authier F, Metioui M, Fabrega S, Kouach M, Briand G: Endosomal proteolysis of internalized insulin at the c-terminal region of the $b$ chain by cathepsin $d$. J Biol Chem 2002;277:9437-9446.

58 Coleman RA, Lewin TM, Van Horn CG, Gonzalez-Baro MR: Do long-chain acylcoa synthetases regulate fatty acid entry into synthetic versus degradative pathways? J Nutr 2002;132:2123-2126. Yahagi N, Shimano H, Hasty $\mathrm{AH}$, Matsuzaka T, Ide T, Yoshikawa T, Amemiya-Kudo M, Tomita S, Okazaki H, Tamura Y, Iizuka Y, Ohashi K, Osuga J, Harada K, Gotoda T, Nagai R, Ishibashi $\mathrm{S}$, Yamada N: Absence of sterol regulatory element-binding protein-1 (srebp-1) ameliorates fatty livers but not obesity or insulin resistance in lep(ob)/lep(ob) mice. J Biol Chem 2002;277:1935319357.

60 Jungas RL: Role of cyclic-3',5'-amp in the response of adipose tissue to insulin. Proc Natl Acad Sci USA 1966;56:757763. 
-61 Huttunen JK, Steinberg D, Mayer SE: Atp-dependent and cyclic amp-dependent activation of rat adipose tissue lipase by protein kinase from rabbit skeletal muscle. Proc Natl Acad Sci USA 1970;67:290-295.

62 Fritz-Wolf K, Schnyder T, Wallimann T, Kabsch W: Structure of mitochondrial creatine kinase. Nature 1996;381:341345 .

63 Miller RE, Hackenberg R, Gershman H: Regulation of glutamine synthetase in cultured 3t3-11 cells by insulin, hydrocortisone, and dibutyryl cyclic amp. Proc Natl Acad Sci USA 1978;75:1418-1422.

64 Gounarides JS, Korach-Andre M, Killary K, Argentieri G, Turner O, Laurent D: Effect of dexamethasone on glucose tolerance and fat metabolism in a dietinduced obesity mouse model. Endocrinology 2008;149:758-766.

65 Ohlson LO, Larsson B, Bjorntorp P, Eriksson H, Svardsudd K, Welin L, Tibblin G, Wilhelmsen L: Risk factors for type 2 (non-insulin-dependent) diabetes mellitus. Thirteen and one-half years of follow-up of the participants in a study of swedish men born in 1913 . Diabetologia 1988;31:798-805.
66 Gorin E, Tal-Or Z, Shafrir E: Glyceroneogenesis in adipose tissue of fasted, diabetic and triamcinolone treated rats. Eur J Biochem 1969;8:370-375.

67 Vaughan CK, Gohlke U, Sobott F, Good VM, Ali MM, Prodromou C, Robinson CV, Saibil HR, Pearl LH: Structure of an hsp90-cdc37-cdk4 complex. Mol Cell 2006;23:697-707.

-68 Xi W, Satoh H, Kase H, Suzuki K, Hattori Y: Stimulated hsp90 binding to enos and activation of the pi3-akt pathway contribute to globular adiponectininduced no production: Vasorelaxation in response to globular adiponectin. Biochem Biophys Res Commun 2005;332:200-205.

69 Cherbavaz DB, Lee ME, Stroud RM, Koshland DE Jr: Active site water molecules revealed in the 2.1 a resolution structure of a site-directed mutant of isocitrate dehydrogenase. J Mol Biol 2000;295:377-385.
Ceccarelli C, Grodsky NB, Ariyaratne N, Colman RF, Bahnson BJ: Crystal structure of porcine mitochondrial nadp+dependent isocitrate dehydrogenase complexed with $\mathrm{mn} 2+$ and isocitrate. Insights into the enzyme mechanism. J Biol Chem 2002;277:43454-43462.

Mercader J, Granados N, Caimari A, Oliver P, Bonet ML, Palou A: Retinolbinding protein 4 and nicotinamide phosphoribosyltransferase/visfatin in rat obesity models. Horm Metab Res 2008;40:467-472.

72 Alberts AW, Strauss AW, Hennessy S, Vagelos PR: Regulation of synthesis of hepatic fatty acid synthetase: Binding of fatty acid synthetase antibodies to polysomes. Proc Natl Acad Sci USA 1975;72:3956-3960.

73 Metskas LA, Kulp M, Scordilis SP: Gender dimorphism in the exercise-naive murine skeletal muscle proteome. Cell Mol Biol Lett 2010;15:507-516. 